\title{
New Approaches for the Synthesis, Cytotoxicity and Toxicity of Heterocyclic Compounds Derived from 2-Cyanomethylbenzo[c]imidazole
}

\author{
Rafat M. Mohareb, ${ }^{1, *}$ Abeer A. Mohamed ${ }^{2}$ and Amira E. M. Abdallah ${ }^{3}$ \\ ${ }^{1}$ Department of Chemistry, Faculty of Science, Cairo University, Giza, A. R. Egypt \\ ${ }^{2}$ National Organization for Research \& Control of Biologicals, Giza, Egypt \\ ${ }^{3}$ Department of Chemistry, Faculty of Science, Helwan University, Cario, Egypt \\ *Corresponding author: E-mail: raafat_mohareb@yahoo.com
}

Received: 04-05-2015

\begin{abstract}
The reaction of ethyl cyanoacetate with $o$-phenylenediamine gave the 2-cyanomethylbenzo[c]imidazole (1). The latter compound was used as the key starting material to synthesise biologically active heterocyclic derivatives. Thus, the reaction of 1 with cyclohexanone and either of benzaldehyde, 4-methoxybenzaldehyde or 4-chlorobenzaldehyde gave the annulated derivatives $\mathbf{2 a}-\mathbf{c}$, respectively. The antitumor evaluations of the newly synthesized products against the three cancer cell lines MCF-7 (breast adeno-carcinoma), NCI-H460 (non-small cell lung cancer) and SF-268 (CNS cancer) showed that compounds $2 \mathbf{b}, \mathbf{6}, \mathbf{1 1 b}, \mathbf{1 1 c}, \mathbf{1 2 b}, \mathbf{1 6 a}, \mathbf{1 6}$ and $18 \mathrm{a}$ exhibited optimal cytotoxic effect against cancer cell lines, with $\mathrm{IC}_{50}$ values in the $\mathrm{nM}$ range. Bioactive compounds are often toxic to shrimp larvae. Thus, in order to monitor these chemicals in vivo lethality to shrimp larvae (Artemia salina), Brine-Shrimp Lethality Assay was used. Compounds $\mathbf{1 1 b}, \mathbf{1 2 b}$ and $\mathbf{1 6 b}$ showed no toxicity against the tested organisms.
\end{abstract}

Keywords: benzimidazole, thiophene, thiazole, synthesis, anti-tumor, toxicity

\section{Introduction}

In recent years benzimidazole derivatives have provided a large number of biologically active compounds that have been intensively used in medicinal chemistry as drugs. They are structural isosteres of naturally occurring nucleotides, which allow them to interact easily with the biopolymers of the living systems and various kinds of biological activities have been obtained. Some 2-aminobenzimidazoles display an appreciable antimicrobial effect. Their corresponding carbamate derivatives have been synthesized for their significant in vivo antifilarial activity. ${ }^{1}$ Concerning the high affinity that they display towards a variety of enzymes and protein receptors, they could be considered as pivotal structures in drug design. ${ }^{2}$ Optimization of benzimidazole-based structures has resulted in marketed drugs, e.g. Omeprazole $^{3}$ and Pimobendan ${ }^{4}$ that are therapeutically useful in the management of peptic ulcer and congestive heart failure, respectively. Many derivatives of benzimidazoles are well known for their antimicrobial, ${ }^{5-10}$ anthelmintic, ${ }^{11}$ antiviral, ${ }^{12-16}$ and antifungal ${ }^{17,18}$ activities. Since 1985 benzimidazole containing compounds have been reported as well known anticancer agents. ${ }^{19-25}$ The role of mammalian DNA topoisomerases as molecular targets for anticancer drugs has been recognized. Some benzimidazoles have been reported as topoisomerase inhibitors, e.g. Hoechst 33258 and Hoechst 33342 (Fig. 1). ${ }^{26,27}$ As the extension of this work, head to head bisbenzimidazole compounds proved high efficacy as DNA binders. ${ }^{28}$ Some widely used anticancer drugs such as RAF265 (CHIR-265; Novartis Pharmaceuticals, Basel, Switzerland) and AZD6244 (ARRY-142886; AstraZeneca, London, England) are known to contain benzimidazole moiety. RAF265 resulted in a reduction in tumor cell growth and in tumor cell apoptosis. ${ }^{29}$ 


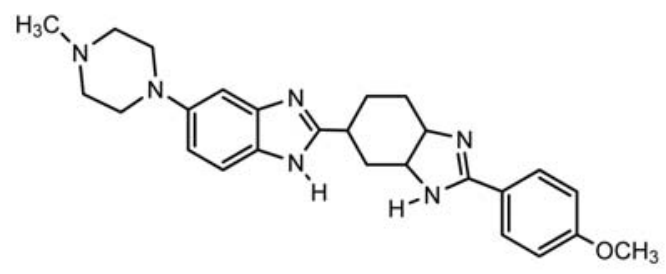

Hoechst 33342

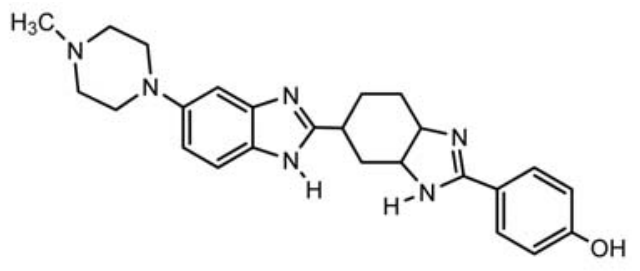

Hoechst 33258

Fig. 1. Examples of topoisomerase inhibitors containing benzimidazole nucleus.

\section{Results and Discussion}

\section{1. Chemistry}

The 2-cyanomethylbenzo[c]imidazole (1) obtained from the reaction of ethyl cyanoacetate with $o$-phenylene- diamine was used as the key starting material to synthesise biologically active heterocyclic derivatives. Thus, the reaction of $\mathbf{1}$ with cyclohexanone and any of benzaldehyde, 4-methoxybenzaldehyde or 4-chlorobenzaldehyde gave the annulated derivatives $\mathbf{2 a - c}$, respectively. The analy-<smiles>N#CCc1nc2ccccc2[nH]1</smiles><smiles>[X]c1ccc(-c2c3c(c(C#N)c4nc5ccccc5n24)CCCC3)cc1</smiles><smiles>Cc1cc(Nc2ccccc2)c(C#N)c2nc3ccc(CC(C)(C)C)cc3n12</smiles>

3<smiles>Cc1cc([NH+]c2ccccc2)c(C#N)c2nc3ccccc3n12</smiles>

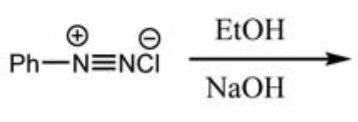<smiles>CC1/C(=N\c2ccccc2)C(=N)C(C#N)c2nc3ccccc3n21</smiles>

4

Scheme 1. 
tical and spectral data of 2a-c were consistent with their respective structures. Thus, the ${ }^{1} \mathrm{H}$ NMR spectrum of $\mathbf{2 a}$ (as an example) showed 2.49-2.88 ( $\mathrm{CH}_{2}$-cyclohexanone), 7.13-8.01 (m, 9H, $\left.\mathrm{C}_{6} \mathrm{H}_{4}, \mathrm{C}_{6} \mathrm{H}_{5}\right)$. Moreover, the ${ }^{13} \mathrm{C} \mathrm{NMR}$ data revealed 38.6, 39.0, 40.2, $40.6\left(4 \times \mathrm{CH}_{2}\right.$-cyclohexanone), $116.2(\mathrm{CN}), 120.3,122.8,124.9,127.8,128.0$, $129.8,131.2,132.6,134.5,134.8,144.3,146.8,150.4(2 \times$ $\mathrm{C}_{6} \mathrm{H}_{4}$, pyridine $\left.\mathrm{C}\right), 164.8(\mathrm{C}=\mathrm{N})$.

The reaction of 1 with acetoacetanilide gave the benzo $[c]$ pyrazolo $[3,2-a]$ pyridine derivative 3 . The latter compound reacted with benzene diazonium chloride to give the phenylazo derivative 4 (Scheme 1). On the other hand, the reaction of $\mathbf{1}$ with acetic acid/acetic anhydride mixture gave the $\mathrm{N}$-acetyl derivative $\mathbf{5}$. Compound $\mathbf{5}$ readily underwent bromination when treated with bromine in acetic acid solution at $60^{\circ} \mathrm{C}$ to give the $N$ - $\alpha$-bromoacetylbenzo $[c]$ imidazole derivative $\mathbf{6}$. The latter compound as $\alpha$-bromocarbonyl compound showed interesting chemical reactivity when treated with some chemical reagents. Thus, the reaction of $\mathbf{6}$ with potassium cyanide gave the benzo[c]imidazo[2,3- $b]$ pyridine derivative 7 . On the other hand, the reaction of 6 with hydrazine hydrate afforded the hydrazine de-<smiles>CC(CCc1nc2ccccc2[nH]1)C(C)(C)C</smiles>

1<smiles>CC(=O)n1c(CC#N)nc2ccccc21</smiles>

5<smiles>CC(=O)n1c(CC#N)nc2ccccc21</smiles>

5
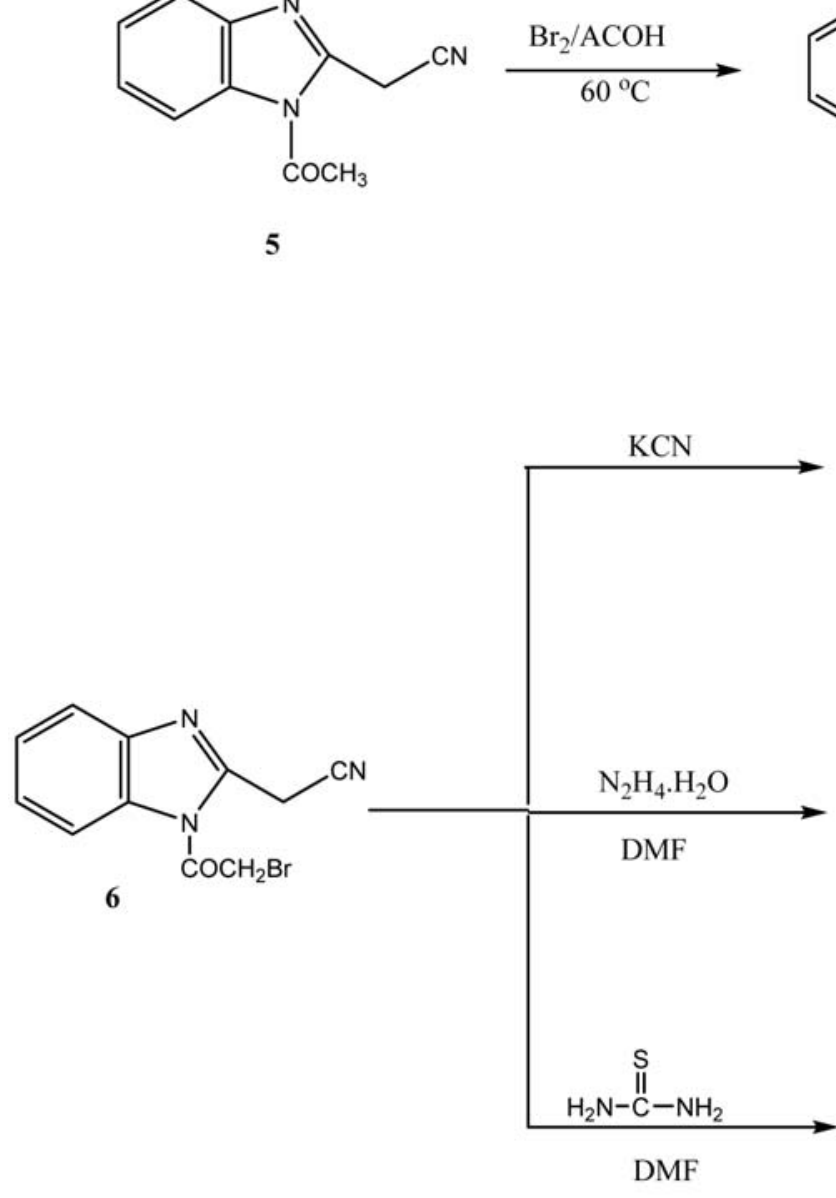<smiles>N#Cc1c(N)cc(O)n2c1nc1ccccc12</smiles>

7<smiles>N#CCc1nc2ccccc2n1C(=O)ON</smiles>

8<smiles>N#CCc1nc2ccccc2n1-c1csc(N)n1</smiles>

9

Scheme 2. 
rivative 8. Compound 6 reacted with thiourea in ethanol to give the thiazole derivative $\mathbf{9}$ (Scheme 2).

Recently, our research group was involved in a comprehensive program involving the reaction of active methylene reagents with phenylisothiocyanate in basic $(\mathrm{KOH})$ dimethylformamide to form the intermediate potassium sulphide salt. The latter undergoes heterocyclization when reacted with $\alpha$-halocarbonyl compounds to give either thiophene or thiazole derivatives depending on the nature of the $\alpha$-halocarbonyl compound and the reaction conditions. ${ }^{30-32}$ Thus, the reaction of 5 with phenylisothiocyanate in $\mathrm{DMF} / \mathrm{KOH}$ solution gave the intermediate potassium sulphide salt $\mathbf{1 0}$. The latter intermediate underwent heterocyclization when reacted with any of $\alpha$ chloroacetone, ethyl chloroacetate or $\alpha$-bromoacetophenone to give the thiophene derivatives $11 a-c$, respectively. The analytical and spectral data of $11 \mathbf{a}-\mathbf{c}$ are consistent with their respective structures.<smiles>CC(=O)n1c(CC#N)nc2ccccc21</smiles>

11a-c<smiles>O[Mg]O</smiles><smiles>CC[AlH2]</smiles><smiles>C[14CH2][14CH2]</smiles><smiles>[X]CC([R])=O</smiles>

a, $\mathrm{X}=\mathrm{Cl}, \mathrm{R}=\mathrm{CH}_{3}$

b, $\mathrm{X}=\mathrm{Cl}, \mathrm{R}=\mathrm{OEt}$ c, $\mathrm{X}=\mathrm{Br}, \mathrm{R}=\mathrm{Ph}$

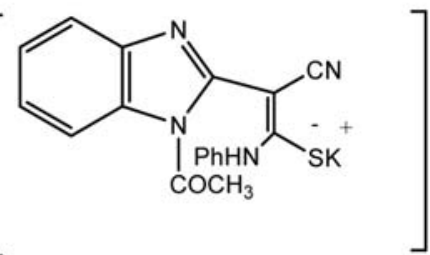

10

\begin{tabular}{|c|c|}
\hline $\mathbf{1 1}$ & $\mathrm{R}$ \\
\hline $\mathbf{a}$ & $\mathrm{CH}_{3}$ \\
\hline $\mathbf{b}$ & $\mathrm{OEt}$ \\
\hline $\mathbf{c}$ & $\mathrm{Ph}$ \\
\hline
\end{tabular}<smiles>CC(=O)n1c(CC#N)nc2ccccc21</smiles>

5<smiles>[X]c1ccccc1C=O</smiles>

$\mathrm{X}=\mathrm{H}$ $\mathrm{X}=\mathrm{OH}$<smiles></smiles>

12a, $X=H$

b, $\mathrm{X}=\mathrm{OH}$<smiles>[X]C1=C(N)C(C#N)=C2Nc3ccccc3N2C1C</smiles>

13a, $\mathrm{X}=\mathrm{CN}$

b, $\mathrm{X}=\mathrm{COOEt}$

Scheme 3. 
The reaction of $\mathbf{5}$ with either benzaldehyde or salicylaldehyde gave the benzylidene derivatives 12a and $\mathbf{1 2 b}$, respectively. On the other hand, the reaction of $\mathbf{5}$ with either malononitrile or ethyl cyanoacetate in DMF containing triethylamine gave the 1,5-dihydrobenzo[4,5]imidazo[1,2-a]pyridine derivatives 13a and 13b, respectively (Scheme 3).

Compound 5 reacted with acetophenone in an oil bath at $120{ }^{\circ} \mathrm{C}$ to give the Knoevenagel condensation pro- duct 14. The latter compound reacted with benzaldehyde to give the benzylidene derivative $\mathbf{1 5}$.

The reactivity of $\mathbf{5}$ towards the well-known Gewald's thiophene synthesis was studied to give biologically active thiophene derivatives. Thus, the reaction of $\mathbf{5}$ with elemental sulfur and either of malononitrile or ethyl cyanoacetate gave the thiophene derivatives $16 \mathbf{a}$ and $\mathbf{1 6 b}$, respectively. On the other hand, the reaction of $\mathbf{5}$ with benzenediazonium chloride in ethanol/sodium hydroxide solution affor-<smiles>CC(=O)OC(C)=C(C)c1nc2ccccc2n1C(C)=O</smiles>

5

14<smiles>CC(=O)OC(C)=C(C#N)c1nc2ccccc2n1C(C)=O</smiles>

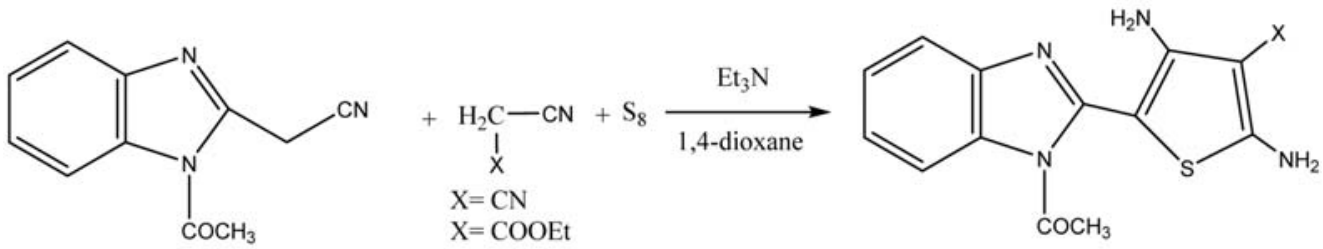<smiles>CC(=O)n1c(CC#N)nc2ccccc21</smiles>

5<smiles>CC(=O)n1c(C(=N)N=N)nc2ccccc21</smiles>

17

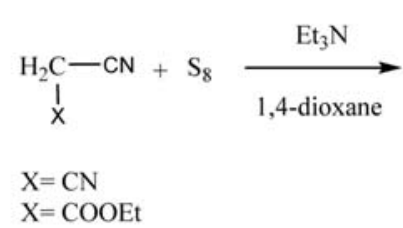

$\mathrm{X}=\mathrm{COOE} \mathrm{t}$<smiles>CC(=O)n1c(C(C#N)=Nc2ccccc2)nc2ccccc21</smiles>

17

Scheme 4. 
ded the phenylhydrazo derivative $\mathbf{1 7}$. Compound $\mathbf{1 7}$ underwent the Gewald's thiophene synthesis through the $\mathrm{N}$ acetyl moiety when reacted with elemental sulfur and either of malononitrile or ethyl cyanoacetate in 1,4-dioxane containing triethylamine under reflux to give the thiophene derivatives 18a and 18b, respectively (Scheme 4).

\section{Anti-tumor and Normal Cell Line Activity Tests}

\section{1. Chemicals}

Reagents: Fetal bovine serum (FBS) and L-glutamine were from Gibco Invitrogen Co. (Scotland, UK). RPMI-1640 medium was from Cambrex (New Jersey, USA). Dimethyl sulfoxide (DMSO), doxorubicin, penicillin, streptomycin and sulforhodamine B (SRB) were from Sigma Chemical Co. (Saint Louis, USA).

\section{1. 1. Cell Cultures}

Three human tumor cell lines, MCF-7 (breast adenocarcinoma), NCI-H460 (non-small cell lung cancer), and SF-268 (CNS cancer) were used. MCF-7 was obtained from the European Collection of Cell Cultures (ECACC, Salisbury, UK), NCI-H460, SF-268 and normal fibroblast were grown as monolayer and routinely maintained in RPMI-1640 medium supplemented with 5\% heat inactivated FBS, 2 mM glutamine and antibiotics (penicillin $100 \mathrm{U} / \mathrm{mL}$, streptomycin $100 \mu \mathrm{g} / \mathrm{mL}$ ), at $37^{\circ} \mathrm{C}$ in a humidified atmosphere containing $5 \% \mathrm{CO}_{2}$. Exponentially growing cells were obtained by plating $1.5 \times 10^{5}$ cells $/ \mathrm{mL}$ for MCF-7 and SF-268 and $0.75 \times 10^{4}$ cells $/ \mathrm{mL}$ for NCIH460, followed by $24 \mathrm{~h}$ of incubation. The effect of the vehicle solvent (DMSO) on the growth of these cell lines was evaluated in all the experiments by exposing untreated control cells to the maximum concentration $(0.5 \%)$ of DMSO used in each assay.

\section{1. 2. Tumor Cell Growth Assay}

The effects of $\mathbf{2 a - c}$ to $\mathbf{1 8 a}, \mathbf{b}$ on the in vitro growth of human tumor cell lines were evaluated according to the procedure adopted by the National Cancer Institute (NCI, USA) in the žIn vitro Anticancer Drug Discovery Screen' that uses the protein-binding dye sulforhodamine B to assess cell growth. ${ }^{33}$ Briefly, exponentially, cells growing in

Table 1. Effect of newly synthesized compounds on the growth of three human tumor cell lines

\begin{tabular}{|c|c|c|c|c|}
\hline \multirow{2}{*}{ Compound } & \multicolumn{4}{|c|}{$\mathrm{GI}_{50}\left(\mu \mathrm{mol} \mathrm{L}{ }^{-1}\right)$} \\
\hline & MCF-7 & NCI-H460 & SF-268 & WI 38 \\
\hline $2 \mathbf{2 a}$ & $33.0 \pm 1.4$ & $20.8 \pm 4.3$ & $20.3 \pm 2.8$ & $38.4 \pm 2.90$ \\
\hline $2 b$ & $0.8 \pm 0.04$ & $0.5 \pm 0.02$ & $0.06 \pm 0.001$ & $20.0 \pm 4.94$ \\
\hline $2 c$ & $22.1 \pm 10.4$ & $30.8 \pm 10.8$ & $26.1 \pm 2.8$ & $28.2 \pm 0.8$ \\
\hline 3 & $33.6 \pm 10.2$ & $40.0 \pm 8.6$ & $38.6 \pm 8.08$ & $>100$ \\
\hline 4 & $32.2 \pm 3.6$ & $36.3 \pm 12.5$ & $40.6 \pm 8.8$ & $50.7 \pm 8.2$ \\
\hline 5 & $22.8 \pm 8.30$ & $22.8 \pm 4.32$ & $22.8 \pm 6.23$ & $44.8 \pm 6.0$ \\
\hline 6 & $0.01 \pm 0.001$ & $0.02 \pm 0.004$ & $0.06 \pm 0.002$ & $>100$ \\
\hline 7 & $28.4 \pm 5.8$ & $22.7 \pm 8.2$ & $30.4 \pm 2.4$ & $18.6 \pm 4.0$ \\
\hline 8 & $23.55 \pm 4.06$ & $34.6 \pm 12.06$ & $45.41 \pm 2.16$ & $>100$ \\
\hline 9 & $33.6 \pm 8.5$ & $40.3 \pm 12.3$ & $30.4 \pm 2.8$ & $62.2 \pm 2.0$ \\
\hline $11 a$ & $26.4 \pm 2.10$ & $12.42 \pm 3.01$ & $10.63 \pm 2.83$ & $>100$ \\
\hline $11 b$ & $0.81 \pm 0.04$ & $0.52 \pm 0.04$ & $0.08 \pm 0.006$ & $40.0 \pm 1.3$ \\
\hline $11 \mathrm{c}$ & $1.6 \pm 0.4$ & $0.6 \pm 0.16$ & $1.8 \pm 0.06$ & $22.4 \pm 1.6$ \\
\hline $12 a$ & $26.2 \pm 2.4$ & $28.6 \pm 2.8$ & $26.8 \pm 8.5$ & $30.2 \pm 2.6$ \\
\hline $12 b$ & $0.02 \pm 0.001$ & $0.03 \pm 0.006$ & $0.06 \pm 0.008$ & $>100$ \\
\hline 13a & $30.22 \pm 6.12$ & $28.99 \pm 4.70$ & $10.39 \pm 6.80$ & $>100$ \\
\hline $13 b$ & $12.6 \pm 2.01$ & $18.6 \pm 6.06$ & $30.4 \pm 2.36$ & $30.6 \pm 10.2$ \\
\hline 14 & $12.33 \pm 2.16$ & $16.36 \pm 2.26$ & $18.20 \pm 5.28$ & $55.5 \pm 8.3$ \\
\hline 15 & $30.7 \pm 6.2$ & $38.5 \pm 6.4$ & $37.5 \pm 8.0$ & $66.0 \pm 18.4$ \\
\hline $16 \mathbf{a}$ & $2.6 \pm 2.8$ & $6.6 \pm 2.2$ & $5.0 \pm 1.81$ & $0.5 \pm 5.1$ \\
\hline $16 b$ & $0.06 \pm 0.006$ & $0.06 \pm 0.006$ & $0.02 \pm 0.008$ & $>100$ \\
\hline 17 & $38 \pm 4.18$ & $39.03 \pm 8.01$ & $22.59 \pm 4.01$ & $20.20 \pm 8.2$ \\
\hline $18 \mathrm{a}$ & $0.08 \pm 0.002$ & $0.08 \pm 0.003$ & $0.02 \pm 0.002$ & $>100$ \\
\hline $18 b$ & $36.0 \pm 7.3$ & $26.7 \pm 2.8$ & $30.4 \pm 2.9$ & $32.6 \pm 6.4$ \\
\hline Doxorubicin & $0.04 \pm 0.008$ & $0.09 \pm 0.008$ & $0.09 \pm 0.007$ & $>100$ \\
\hline
\end{tabular}

Results are given in concentrations that were able to cause $50 \%$ of cell growth inhibition $\left(\mathrm{GI}_{50}\right)$ after a continuous exposure of $48 \mathrm{~h}$ and show means \pm SEM of three-independent experiments performed in duplicate. 
96-well plates were then exposed for $48 \mathrm{~h}$ to five serial concentrations of each compound, starting from a maximum concentration of $150 \mu \mathrm{M}$. Following this exposure period adherent cells were fixed, washed, and stained. The bound stain was solubilized and the absorbance was measured at $492 \mathrm{~nm}$ in a plate reader (Bio-Tek Instruments Inc., Power wave XS, Winooski, USA). For each test compound and cell line, a dose-response curve was obtained and the growth inhibition of 50\% $\left(\mathrm{GI}_{50}\right)$, corresponding to the concentration of the compounds that inhibited $50 \%$ of the net cell growth was calculated as described elsewhere. Doxorubicin was used as a positive control and tested in the same manner.

\section{1. 3. Structure Activity Relationship:}

From Table 1 it is clear that the benzimidazole moiety was found to be crucial for the cytotoxic effect of the cyclic compounds 2a-c to 18a,b. Compounds $2 \mathbf{b}, \mathbf{6}, \mathbf{1 1 b}, 11 \mathrm{c}$, 12b, 16a, 16b and 18a exhibited optimal cytotoxic effect against cancer cell lines, with $\mathrm{IC}_{50}$ values in the $\mathrm{nM}$ range. Comparing the cytotoxicity of the benzimidazothiophenes 11b and 11c, it is obvious that the cytotoxicity of $\mathbf{1 1 b}$ is higher than that of 11c. The presence of the 2-EtO group in 11b is responsible for its high potency. Considering the 7,8,9,10-tetrahydrobenzo[4,5]imidazo[1,2- $b$ ]isoquinoline derivatives $\mathbf{2 a - c}$, it is clear that the cytotoxicity of $\mathbf{2 b}$ is higher than those of $\mathbf{2 a}$ and $\mathbf{2 c}$. Such high cytotoxicity of $\mathbf{2 b}$ is attributed to the presence of the 4-chlorophenylisoquinoline moiety together with the benzimidazole moiety. The high cytotoxicity of $\mathbf{1 6 b}$ relative to $\mathbf{1 6 a}$ is also explained in terms of the presence of the 3-EtO moiety. On the other hand, by considering the ( $1 H$-benzo[ $d]$ imidazol-1-yl)-2-aminothiophene derivatives 18a and $\mathbf{1 8 b}$ it is clear that the presence of the 2-carbonitrile group present in 18a is responsible for its high potency. The bromo- $1 H$-benzo[ $d]$ imidazole derivative 6 showed the maximum cytotoxicity effect towards the three cancer cell lines followed by acetyl- $1 H$-benzo $[d]$ imidazolhydroxyphenyl derivative $\mathbf{1 2 b}$.

\section{2. Toxicity}

Bioactive compounds are often toxic to shrimp larvae. Thus, in order to monitor these chemicals, in vivo lethality to shrimp larvae (Artemia salina), Brine-Shrimp Lethality Assay ${ }^{34}$ was used. Results were analyzed with $\mathrm{LC}_{50}$ program to determine $\mathrm{LC}_{50}$ values and $95 \%$ confidence intervals. ${ }^{35}$ Results are given in Table 2 for the compounds which exhibited optimal cytotoxic effect against cancer cell lines, being the eight compounds $2 \mathbf{b}, \mathbf{6}, \mathbf{1 1 b}, \mathbf{1 1 c}, \mathbf{1 2 b}, \mathbf{1 6}$, $\mathbf{1 6} \mathbf{b}$ and 18a. The shrimp lethality assay is considered as a useful tool for preliminary assessment of toxicity, and it has

Table 2. Toxicity of the most potent compounds against the cancer cell lines

\begin{tabular}{|c|c|c|c|c|c|c|}
\hline Compound & Conc. $(\mu \mathrm{g} / \mathrm{mL})$ & Mortality $^{\mathbf{a}}$ & Toxicity & $\mathbf{L C}_{50}$ & Upper 95\% lim & Lower $95 \%$ lim \\
\hline \multirow[t]{3}{*}{$\overline{2 b}$} & 10 & 6 & Very toxic & 12.05 & - & - \\
\hline & 100 & 8 & & & & \\
\hline & 1000 & 10 & & & & \\
\hline \multirow[t]{3}{*}{$\overline{6}$} & 10 & 1 & Very toxic & 18.38 & - & - \\
\hline & 100 & 6 & & & & \\
\hline & 1000 & 10 & & & & \\
\hline \multirow[t]{3}{*}{$\overline{11 b}$} & 10 & 0 & Non toxic & 982.15 & - & - \\
\hline & 100 & 0 & & & & \\
\hline & 1000 & 2 & & & & \\
\hline \multirow[t]{3}{*}{$\overline{11 c}$} & 10 & 0 & Harmful & 420.28 & 112.23 & 90.55 \\
\hline & 100 & 5 & & & & \\
\hline & 1000 & 10 & & & & \\
\hline \multirow[t]{3}{*}{$\overline{12 b}$} & 10 & 0 & Non toxic & 880.42 & - & - \\
\hline & 100 & 1 & & & & \\
\hline & 1000 & 4 & & & & \\
\hline \multirow[t]{3}{*}{ 16a } & 10 & 2 & Very toxic & 14.88 & - & - \\
\hline & 100 & 8 & & & & \\
\hline & 1000 & 10 & & & & \\
\hline \multirow[t]{3}{*}{$\overline{16 b}$} & 10 & 0 & Non toxic & 999.33 & - & - \\
\hline & 100 & 0 & & & & \\
\hline & 1000 & 5 & & & & \\
\hline \multirow[t]{3}{*}{$\overline{18 a}$} & 10 & 0 & Harmful & 22.70 & 210.59 & 160.22 \\
\hline & 100 & 6 & & & & \\
\hline & 1000 & 8 & & & & \\
\hline
\end{tabular}

\footnotetext{
${ }^{\text {a }}$ Ten organisms (A. salina) tested for each concentration.
} 
been used for the detection of fungal toxins, plant extract toxicity, heavy metals, cyano bacteria toxins, pesticides, and cytotoxicity testing of dental materials, ${ }^{36}$ natural and synthetic organic compounds. ${ }^{34}$ It has also been shown that A. salina toxicity test results have a correlation with rodent and human acute oral toxicity data. Generally, a good correlation was obtained between $A$. salina toxicity test and the rodent data. Likewise, the predictive screening potential of the aquatic invertebrate tests for acute oral toxicity in man, including $A$. salina toxicity test, was slightly better than the rat test for test compounds. ${ }^{37}$

In order to prevent the toxicity results from possible false effects originated from solubility of compounds and DMSO's possible toxicity effect, compounds were prepared by dissolving in DMSO in the suggested DMSO volume ranges. It is clear from Table 2 that $\mathbf{1 1 b}, \mathbf{1 2 b}$, and $\mathbf{1 6 b}$ showed no toxicity against the tested organisms. On the other hand, 2b, $\mathbf{6}$ and 16a are very toxic, in addition, 11c and $\mathbf{1 8 a}$ are harmful.

\section{2. 1. Toxicity Method}

All toxicity tests were 96-h static renewal tests and water quality measurements (dissolved oxygen, $\mathrm{pH}$, temperature, salinity) were taken in the control containers each day. Tests were run in a Revcos Environmental Chamber at $25{ }^{\circ} \mathrm{C}, 20 \%$ salinity, and a 16-h light : 8-h dark cycle. A media change was made every $24 \mathrm{~h}$. Larvae used for all tests were one to two days old and exposed in 600-mL glass beakers containing $400 \mathrm{~mL}$ of media with 10 larvae/beaker and three replicates/concentration. Larvae were fed newly hatched Artemia after daily media change. The concentration of each compound was taken in terms 10, 100 and $1000 \mathrm{mg} / \mathrm{mL}$. Adult shrimp toxicity tests were also run to complete the grass shrimp toxicity profile. Adult shrimp (acclimated for two weeks before testing) were exposed in 4-L wide-mouth glass jars containing $2 \mathrm{~L}$ of media and 10 shrimp/jar with two replicates/concentration and were run under conditions as described above for larvae. ${ }^{38}$

\section{Experimental}

All melting points are uncorrected. IR spectra were recorded on $\mathrm{KBr}$ discs on a PyeUnicam SP-1000 spectrophotometer. ${ }^{1} \mathrm{H}$ and ${ }^{13} \mathrm{C}$ NMR spectra were measured on a Varian EM-390-200 MHz in DMSO as solvent using TMS as internal standard, and chemical shifts are expressed as $\delta$. Analytical data were obtained from the Microanalytical Data Unit at Cairo University, Giza, Egypt.

\section{General procedure for the synthesis of the imidazo [1,2-b]isoquinolines 2a-c}

To a solution of $1(1.57 \mathrm{~g}, 10 \mathrm{mmol})$ in ethanol $(25$ $\mathrm{mL})$ containing triethylamine $(0.5 \mathrm{~mL})$, cyclohexanone
$(0.98 \mathrm{~g}, 10 \mathrm{mmol})$ and any of benzaldehyde $(1.06 \mathrm{~g}, 10$ mmol), 4-chlorobenzaldehyde $(1.40 \mathrm{~g}, 10 \mathrm{mmol})$ or 4 methoxybenzaldehyde $(1.36 \mathrm{~g}, 10 \mathrm{mmol})$ were added. The reaction mixture was heated under reflux for $3 \mathrm{~h}$, then poured into a beaker containing ice/water mixture containing a few drops of hydrochloric acid. The solid product formed was collected by filtration and dried. The obtained product was crystallized from ethanol to give greenish brown crystals.

\section{1-Phenyl-7,8,9,10-tetrahydrobenzo[4,5]imidazo[1,2- b]isoquinoline-6-carbonitrile (2a).}

Yield $2.26 \mathrm{~g} \mathrm{(70 \% );} \mathrm{m.p.} 223-225^{\circ} \mathrm{C}$; Anal. Calcd. for $\mathrm{C}_{22} \mathrm{H}_{17} \mathrm{~N}_{3}$ (323.39): C, 81.71; H, 5.30; N, 12.99\%; found: $\mathrm{C}$, 81.60; 4.85; N, 13.20\%. IR (KBr) $v / \mathrm{cm}^{-1} 3092-3030(\mathrm{CH}$ aromatic), $2887(\mathrm{CH}$-alph.), $2222(\mathrm{CN}), 1523(\mathrm{C}=\mathrm{N}), 1589$, $1437(\mathrm{C}=\mathrm{C}) .{ }^{1} \mathrm{H}$ NMR (DMSO- $\left.d_{6}\right) \delta 2.49-2.88(\mathrm{~m}, 8 \mathrm{H}$, 4 $\mathrm{CH}_{2}$-cyclohexanone), 7.13-8.01 (m, 9H, $\left.\mathrm{C}_{6} \mathrm{H}_{4}, \mathrm{C}_{6} \mathrm{H}_{5}\right) .{ }^{13} \mathrm{C}$ NMR (DMSO- $\left.d_{6}\right) \delta 38.6,39.0,40.2,40.6\left(4 \times \mathrm{CH}_{2}\right), 116.2$ (CN), 120.3, 122.8, 124.9, 127.8, 128.0, 129.8, 131.2, 132.6, 134.5, 134.8, 144.3, 146.8, $150.4\left(2 \times \mathrm{C}_{6} \mathrm{H}_{4}\right.$, pyridine C), $164.8(\mathrm{C}=\mathrm{N})$. MS $(\mathrm{m} / z) 323\left(\mathrm{M}^{+}, 23 \%\right)$.

\section{1-(4-Chlorophenyl)-7,8,9,10-tetrahydrobenzo[4,5] imidazo[1,2-b]isoquinoline-6-carbonitrile (2b).}

Yield $2.32 \mathrm{~g} \mathrm{(65 \% );} \mathrm{m.p.} 267-269^{\circ} \mathrm{C}$; Anal. Calcd. for $\mathrm{C}_{22} \mathrm{H}_{16} \mathrm{ClN}_{3}$ (357.84): C, 73.84; $\mathrm{H}, 4.51 ; \mathrm{N}, 11.74 \%$; found: C, 73.61; H, 4.38; N, 11.94\%. IR (KBr) v/ $\mathrm{cm}^{-1}$ 3091- 3027 (CH aromatic), 2900 (CH-alph.), $2222(\mathrm{CN})$, 1584, $1488(\mathrm{C}=\mathrm{C}), 1523(\mathrm{C}=\mathrm{N}) .{ }^{1} \mathrm{H}$ NMR $\left(\mathrm{DMSO}-d_{6}\right) \delta$ 2.49-2.51 (m, 8H, 4CH $8 \mathrm{H}, 2 \mathrm{C}_{6} \mathrm{H}_{4}$ ). ${ }^{13} \mathrm{C}$ NMR (DMSO- $\left.d_{6}\right) \delta 38.8,39.12,39.7$, $39.9\left(4 \times \mathrm{CH}_{2}\right), 115.9(\mathrm{CN}), 120.0,122.9,125.8,126.2$, $130.2,131.1,134.5,136.1,138.4,140.2,144.9,145.6$, $150.3\left(2 \times \mathrm{C}_{6} \mathrm{H}_{4}\right.$, pyridine-C $), 165.2(\mathrm{C}=\mathrm{N})$. MS $(\mathrm{m} / \mathrm{z}) 357$ $\left(\mathrm{M}^{+}, 80 \%\right)$.

\section{1-(4-Methoxyphenyl)-7,8,9,10-tetrahydrobenzo[4,5]} imidazo[1,2-b] isoquinoline-6-carbonitrile (2c).

Yield 2.58 g (73\%); m.p. $243-246{ }^{\circ} \mathrm{C}$; Anal. Calcd. for $\mathrm{C}_{23} \mathrm{H}_{19} \mathrm{~N}_{3} \mathrm{O}$ (353.42): C, 78.16; H, 5.42; N, 11.89\%; found: C, 77.33; H, 4.05; N, 11.06\%. IR (KBr) v/ $\mathrm{cm}^{-1}$ 3103-3015 (CH aromatic), 2901-2838 (CH-alph.), 2212 $(\mathrm{CN}), 1643(\mathrm{C}=\mathrm{O}), 1512(\mathrm{C}=\mathrm{N}), 1589,1446(\mathrm{C}=\mathrm{C}) .{ }^{1} \mathrm{H}$ NMR (DMSO- $\left.d_{6}\right) \delta 1.30\left(\mathrm{~s}, 3 \mathrm{H}, \mathrm{CH}_{3}\right), 2.49-2.51(\mathrm{~m}, 8 \mathrm{H}$, $4 \times \mathrm{CH}_{2}$-cyclohexanone), 6.91-8.02 (m, $\left.8 \mathrm{H}, 2 \mathrm{C}_{6} \mathrm{H}_{4}\right) .{ }^{13} \mathrm{C}$ NMR (DMSO- $d_{6}$ ) $\delta 38.55,38.84,39.39,39.67,39.95$ (4- $\mathrm{CH}_{2}$, cyclohexanone), $55.5\left(\mathrm{CH}_{3}\right), 116.7(\mathrm{CN}), 120.3$, $123.6,124.6,125.3,130.8,131.1,134.5,136.6,138.8$, $140.6,143.7,146.8,150.2\left(2 \times \mathrm{C}_{6} \mathrm{H}_{4}\right.$, pyridine-C $), 165.8$ $(\mathrm{C}=\mathrm{N})$. MS $(\mathrm{m} / \mathrm{z}) 353\left(\mathrm{M}^{+}, 36 \%\right)$.

\section{1-Methyl-3-(phenylamino)benzo[4,5]imidazo[1,2-a] pyridine-4-carbonitrile (3).}

To a mixture of $\mathbf{1}(1.57 \mathrm{~g}, 10 \mathrm{mmol})$ and acetoacetanilide $(1.77 \mathrm{~g}, 10 \mathrm{mmol})$, ammonium acetate $(0.77 \mathrm{~g}, 10$ 
mmol) was added. The reaction mixture was heated in oil bath at $140{ }^{\circ} \mathrm{C}$ for $1 \mathrm{~h}$ then left to cool. The semisolid formed was triturated with ethanol $(40 \mathrm{~mL})$ and the formed solid product was collected by filtration and dried. The obtained product was crystallized from ethanol to give light amber crystals.

Yield $2.27 \mathrm{~g} \mathrm{(76 \% );} \mathrm{m.p.} \mathrm{>} 300{ }^{\circ} \mathrm{C}$; Anal. Calcd. for $\mathrm{C}_{19} \mathrm{H}_{14} \mathrm{~N}_{4}$ (298.34): C, 76.49; H, 4.73; N, 18.78\%; found: C, 76.33; H, 4.44; N, 18.63\%. IR (KBr) v/cm ${ }^{-1}$ 3423-3106 (NH), 3056 (CH aromatic), 2955-2829 (CH-alph.), 2208 (CN), 1569, $1489(\mathrm{C}=\mathrm{C}), 1526(\mathrm{C}=\mathrm{N})$. ${ }^{1} \mathrm{H}$ NMR $\left(\mathrm{DMSO}-d_{6}\right) \delta 1.91\left(\mathrm{~s}, 3 \mathrm{H}, \mathrm{CH}_{3}\right), 5.94(\mathrm{~s}, 1 \mathrm{H}$, pyridine $\left.\mathrm{C}_{5}\right), 7.05-7.55\left(\mathrm{~m}, 9 \mathrm{H}, \mathrm{C}_{6} \mathrm{H}_{4}, \mathrm{C}_{6} \mathrm{H}_{5}\right), 13.19(\mathrm{~s}, 1 \mathrm{H}$, $\mathrm{NH}) .{ }^{13} \mathrm{C}$ NMR (DMSO- $\left.d_{6}\right) \delta 20.24\left(\mathrm{CH}_{3}\right), 116.2(\mathrm{CN})$, $111.1,115.9,120.7,120.7,122.1,126.2,127.5,128.7$, $131.5,104.2,146.6,150.8,154.0,155.1\left(\mathrm{C}_{6} \mathrm{H}_{4}, \mathrm{C}_{6} \mathrm{H}_{5}\right.$, pyridine-C), $164.1(\mathrm{C}=\mathrm{N})$. MS $(\mathrm{m} / \mathrm{z}) 298\left(\mathrm{M}^{+}, 18 \%\right)$.

\section{1-Methyl-2-(2-phenylhydrazono)-3-(phenylimino)-1,2, 3,4-tetrahydrobenzo- $[4,5]$ imidazo-[1,2-a]pyridine-4- carbonitrile (4)}

To a cold $\left(0-5{ }^{\circ} \mathrm{C}\right)$ solution of $\mathbf{3}(1.20 \mathrm{~g}, 40.2 \mathrm{mmol})$ in ethanol $(50 \mathrm{~mL})$ containing sodium hydroxide solution $(10 \mathrm{~mL}, 10 \%)$ and a solution of benzenediazonium chloride $(40.2 \mathrm{mmol})$ [which was prepared by dissolving sodium nitrite $(0.60 \mathrm{~g}, 80.4 \mathrm{mmol})$ in water $(2 \mathrm{~mL})$ was added to a cold solution of aniline $(0.4 \mathrm{~mL}, 40.2 \mathrm{mmol})$ containing the appropriate amount of hydrochloric acid with continuous stirring] was added with continuous stirring. The reaction mixture was stirred at room temperature for $3 \mathrm{~h}$ and the solid product formed was collected by filtration and dried. The obtained product was crystallized from ethanol to give red crystals.

Yield $2.67 \mathrm{~g}$ (66\%); m.p. $245-248{ }^{\circ} \mathrm{C}$; Anal. Calcd. for $\mathrm{C}_{25} \mathrm{H}_{20} \mathrm{~N}_{6}$ (404.47): C, 74.24; $\mathrm{H}, 4.98 ; \mathrm{N}, 20.78 \%$; found: C, 74.05; H, 4.88; N, 19.82\%. IR (KBr) v/cm ${ }^{-1}$ 3419-3200 (NH), 3058 (CH aromatic), 2961-2854 (CH-alph.), $2210(\mathrm{CN}), 1600(\mathrm{C}=\mathrm{N}), 1450(\mathrm{C}=\mathrm{C}) .{ }^{1} \mathrm{H}$ NMR (DMSO- $\left.d_{6}\right) \delta 1.91\left(\mathrm{~s}, 3 \mathrm{H}, \mathrm{CH}_{3}\right), 5.82(\mathrm{~s}, 1 \mathrm{H}$, pyridine $\left.\mathrm{C}_{6}\right), 7.17\left(\mathrm{~s}, 1 \mathrm{H}\right.$, pyridine $\left.\mathrm{C}_{3}\right), 7.25-7.79(\mathrm{~m}, 14 \mathrm{H}$, $\left.\mathrm{C}_{6} \mathrm{H}_{4}, 2 \times \mathrm{C}_{6} \mathrm{H}_{5}\right), 8.53(\mathrm{~s}, 1 \mathrm{H}, \mathrm{NH}) .{ }^{13} \mathrm{C} \mathrm{NMR}$ (DMSO- $\left.d_{6}\right) \delta$ $19.9\left(\mathrm{CH}_{3}\right), 115.8(\mathrm{CN}), 118.1,120.7,120.7,120.7$, $121.4,122.0,122.9,123.1,123.9,124.5,124.9,125.83$, $126.1,127.3,128.7,141.3,156.1\left(\mathrm{C}_{6} \mathrm{H}_{4}, 2 \times \mathrm{C}_{6} \mathrm{H}_{5}\right.$, pyridine-C), 173.2, $186.9(2 \times \mathrm{C}=\mathrm{N})$. $\mathrm{MS}(\mathrm{m} / \mathrm{z}) 404\left(\mathrm{M}^{+}, 66 \%\right)$.

\section{2-(1-Acetyl-1H-benzo[d]imidazol-2(3H)-ylidene) ace- tonitrile (5)}

A solution of $1(1.57 \mathrm{~g}, 10 \mathrm{mmol})$ in acetic acid (15 $\mathrm{mL})$ and acetic anhydride $(35 \mathrm{~mL})$ was heated under reflux till a precipitate is formed after $30 \mathrm{~min}$, then poured into a beaker containing ice/water mixture. The solid product formed was collected by filtration and dried. The obtained product was crystallized from ethanol to give gold crystals.

Yield $1.47 \mathrm{~g}(74 \%)$; m.p. $>300{ }^{\circ} \mathrm{C}$; Anal. Calcd. for $\mathrm{C}_{11} \mathrm{H}_{9} \mathrm{~N}_{3} \mathrm{O}$ (199.21): C, 66.32; H, 4.55; N, 21.09\%; found:
C, 64.48; H, 4.33; N, 21.63\%. IR (KBr) v/cm 3069 (CH aromatic), 2965-2871 (CH-alph.), $2192(\mathrm{CN}), 1630$ $(\mathrm{C}=\mathrm{O}), 1594,1410(\mathrm{C}=\mathrm{C}), 1513(\mathrm{C}=\mathrm{N}) .{ }^{1} \mathrm{H}$ NMR (DM$\left.\mathrm{SO}-d_{6}\right) \delta 2.21\left(\mathrm{~s}, 3 \mathrm{H}, \mathrm{CH}_{3}\right), 3.30\left(\mathrm{~s}, 2 \mathrm{H}, \mathrm{CH}_{2}\right), 7.2-7.51$ $\left(\mathrm{m}, 4 \mathrm{H}, \mathrm{C}_{6} \mathrm{H}_{4}\right) .{ }^{13} \mathrm{C}$ NMR (DMSO- $\left.d_{6}\right) \delta 27.0\left(\mathrm{CH}_{3}\right), 65.3$ $\left(\mathrm{CH}_{2}\right), 115.8(\mathrm{CN}), 121.1,123.1,124.0,125.3,126.0$, $130.3\left(\mathrm{C}_{6} \mathrm{H}_{4}\right), 151.1(\mathrm{C}=\mathrm{O}), 189.2(\mathrm{C}=\mathrm{N}) . \mathrm{MS}(\mathrm{m} / \mathrm{z}) 199$ $\left(\mathrm{M}^{+}, 49 \%\right)$.

\section{2-(1-Bromo-1H-benzo[d]imidazol-2-yl)acetonitrile (6)}

A solution of $5(1.99 \mathrm{~g}, 10 \mathrm{mmol})$ in glacial acetic acid $(10 \mathrm{~mL})$ was warmed to $60^{\circ} \mathrm{C}$, then bromine $(0.08 \mathrm{~g}$, $10 \mathrm{mmol})$ in acetic acid $(10 \mathrm{~mL})$ was added drop-wise with continuous stirring. The reaction mixture was stirred for $1.5 \mathrm{~h}$ then poured into ice/water and the solid product formed was collected by filtration. The obtained product was crystallized from ethanol to give brownish orange crystals.

Yield 1.95 g (70\%); m.p. > $300{ }^{\circ} \mathrm{C}$; Anal. Calcd. for $\mathrm{C}_{11} \mathrm{H}_{8} \mathrm{BrN}_{3} \mathrm{O}$ (278.10): C, 47.51; H, 2.90; N, 15.11\%; found: C, 47.33; H, 3.89; N, 13.49\%. IR (KBr) v/ $\mathrm{cm}^{-1}$ 3050 (CH aromatic), 2971 (CH-alph.), $2193(\mathrm{CN}), 1630$ $(\mathrm{C}=\mathrm{O}), 1602,1474(\mathrm{C}=\mathrm{C}) .{ }^{1} \mathrm{H}$ NMR (DMSO- $\left.d_{6}\right) \delta 3.30$, $4.00\left(2 \mathrm{~s}, 4 \mathrm{H}, 2 \times \mathrm{CH}_{2}\right), 7.20-7.63\left(\mathrm{~m}, 4 \mathrm{H}, \mathrm{C}_{6} \mathrm{H}_{4}\right) .{ }^{13} \mathrm{C} \mathrm{NMR}$ $\left(\mathrm{DMSO}-d_{6}\right) \delta 55.8,62.7\left(2 \times \mathrm{CH}_{2}\right), 116.4(\mathrm{CN}), 119.2$, 121.3, 124.8, 129.3, $133.6\left(\mathrm{C}_{6} \mathrm{H}_{4}\right), 168.8(\mathrm{C}=\mathrm{O}), 172.7$ $(\mathrm{C}=\mathrm{N})$. MS $(\mathrm{m} / \mathrm{z}) 278\left(\mathrm{M}^{+}, 28 \%\right)$.

\section{3-Amino-1-hydroxybenzo[4,5]imidazo[1,2-a]pyridine- 4-carbonitrile (7)}

To a solution of 6 ( $2.78 \mathrm{~g}, 10 \mathrm{mmol})$ in dimethylformamide $(5 \mathrm{~mL})$ heated on a water bath at $60^{\circ} \mathrm{C}$ potassium cyanide $(0.65 \mathrm{~g}, 10 \mathrm{mmol})$, dissolved in a least amount of water, was added while stirring. The reaction mixture was left in the water bath for $30 \mathrm{~min}$ at $60^{\circ} \mathrm{C}$ then poured into a beaker containing ice/water mixture and a few drops of hydrochloric acid. The solid product formed was collected by filtration and dried. The obtained product was crystallized from ethanol to give reddish brown crystals.

Yield $1.93 \mathrm{~g}(86 \%)$; m.p. $>300{ }^{\circ} \mathrm{C}$; Anal. Calcd. for $\mathrm{C}_{12} \mathrm{H}_{8} \mathrm{~N}_{4} \mathrm{O}$ (224.22): C, 64.28; H, 3.60; N, 24.99\%; found: $\mathrm{C}, 63.88 ; \mathrm{H}, 3.88 ; \mathrm{N}, 24.69 \%$. IR $(\mathrm{KBr}) \mathrm{v} / \mathrm{cm}^{-1}$ 3436-3233 $\left(\mathrm{OH}, \mathrm{NH}_{2}\right), 3050(\mathrm{CH}$ aromatic), $2193(\mathrm{CN})$, $1513(\mathrm{C}=\mathrm{N}), 1597(\mathrm{C}=\mathrm{C}) .{ }^{1} \mathrm{H}$ NMR (DMSO- $\left.d_{6}\right) \delta 5.21(\mathrm{~s}$, $2 \mathrm{H}, \mathrm{D}_{2} \mathrm{O}$ exchangeable, $\left.\mathrm{NH}_{2}\right), 7.22\left(\mathrm{~s}, 1 \mathrm{H}\right.$, pyridine $\left.\mathrm{C}_{5}\right)$, 7.21-7.88 (m, 4H, $\left.\mathrm{C}_{6} \mathrm{H}_{4}\right), 12.91(\mathrm{~s}, 1 \mathrm{H}, \mathrm{OH}) .{ }^{13} \mathrm{C} \mathrm{NMR}$ $\left(\mathrm{DMSO}-d_{6}\right) \delta 116.8(\mathrm{CN}), 119.2,121.3,124.8,129.3$, 133.6, 136.2, 136.8, 140.1, 142.4, 144.1, $146.7\left(\mathrm{C}_{6} \mathrm{H}_{4}\right)$. MS $(\mathrm{m} / \mathrm{z}) 224\left(\mathrm{M}^{+}, 60 \%\right)$.

\section{2-(1-(2-Hydrazinylacetyl)-1H-benzo[d]imidazol-2-yl) acetonitrile (8)}

To a solution of $6(2.78 \mathrm{~g}, 10 \mathrm{mmol})$ in dimethylformamide $(5 \mathrm{~mL})$ hydrazine hydrate $(0.50 \mathrm{~g}, 10 \mathrm{mmol})$ was added. The reaction mixture was stirred for $3 \mathrm{~h}$ at room temperature then poured into a beaker containing acidi- 
fied ice/water mixture. The solid product formed was collected by filtration and dried. The obtained product was crystallized from ethanol to give light brown crystals.

Yield $2.01 \mathrm{~g}(88 \%)$; m.p. $>300{ }^{\circ} \mathrm{C}$; Anal. Calcd. for $\mathrm{C}_{11} \mathrm{H}_{11} \mathrm{~N}_{5} \mathrm{O}$ (229.24): C, 57.63; H, 4.84; N, 30.55\%; found: C, 57.80; H, 4.69; N, 30.79\%. IR $(\mathrm{KBr}) \mathrm{v} / \mathrm{cm}^{-1}$ 3414-3214 (NH, $\left.\mathrm{NH}_{2}\right), 3100(\mathrm{CH}$ aromatic), 2900 (CH-alph.), $2194(\mathrm{CN}), 1668(\mathrm{C}=\mathrm{O}), 1600,1470(\mathrm{C}=\mathrm{C})$, $1519(\mathrm{C}=\mathrm{N}) .{ }^{1} \mathrm{H}$ NMR (DMSO- $\left.d_{6}\right) \delta 3.34\left(\mathrm{~s}, 2 \mathrm{H}, \mathrm{CH}_{2}\right), 4.40$ (s, 2H, $\mathrm{CH}_{2}$ ), 7.21-7.63 (m, 4H, $\left.\mathrm{C}_{6} \mathrm{H}_{4}\right), 5.76\left(\mathrm{~s}, 2 \mathrm{H}, \mathrm{NH}_{2}\right.$ ), $12.74(\mathrm{~s}, 1 \mathrm{H}, \mathrm{NH}) .{ }^{13} \mathrm{C}$ NMR (DMSO- $\left.d_{6}\right) \delta 56.8,65.9$ $\left(2 \times \mathrm{CH}_{2}\right), 120.8,122.4,126.7,128.3,133.6,145.9\left(\mathrm{C}_{6} \mathrm{H}_{4}\right)$, 164.2 $(\mathrm{C}=\mathrm{O}), 172.8(\mathrm{C}=\mathrm{N})$. MS $(\mathrm{m} / \mathrm{z}) 229\left(\mathrm{M}^{+}, 18 \%\right)$.

\section{2-(1-(2-Aminothiazol-4-yl)-1H-benzo[d]imidazol-2-yl) acetonitrile (9)}

To a solution of $6(2.78 \mathrm{~g}, 10 \mathrm{mmol})$ in dimethylformamide $(20 \mathrm{~mL})$, thiourea $(0.76 \mathrm{~g}, 10 \mathrm{mmol})$ was added. The reaction mixture was heated under reflux for $3 \mathrm{~h}$ then left to cool. The solid product formed was collected by filtration and dried. The obtained product was crystallized from ethanol to give brown crystals.

Yield $2.12 \mathrm{~g} \mathrm{(83 \% );} \mathrm{m.p.}>300{ }^{\circ} \mathrm{C}$; Anal. Calcd. for $\mathrm{C}_{12} \mathrm{H}_{9} \mathrm{~N}_{5} \mathrm{~S}$ (255.30): C, 56.45; H, 3.55; N, 27.43; S, $12.56 \%$; found: C, 56.78; H, 3.69; N, 27.39; S, $12.48 \%$. IR $(\mathrm{KBr}) \mathrm{v} / \mathrm{cm}^{-1} 3428-3227\left(\mathrm{NH}_{2}\right), 3050(\mathrm{CH}$ aromatic), 2967-2922 (CH-alph.), $2195(\mathrm{CN}), 1513(\mathrm{C}=\mathrm{N}), 1597$, $1474(\mathrm{C}=\mathrm{C}) .{ }^{1} \mathrm{H}$ NMR (DMSO- $\left.d_{6}\right) \delta 3.30\left(\mathrm{~s}, 2 \mathrm{H}, \mathrm{CH}_{2}\right)$, $5.21\left(\mathrm{~s}, 2 \mathrm{H}, \mathrm{NH}_{2}\right), 6.23\left(\mathrm{~s}, 1 \mathrm{H}\right.$, thiazole $\left.\mathrm{C}_{5}\right), 7.24-7.63(\mathrm{~m}$, $\left.4 \mathrm{H}, \mathrm{C}_{6} \mathrm{H}_{4}\right) .{ }^{13} \mathrm{C}$ NMR (DMSO-d $)_{6} \delta 55.6,\left(\mathrm{CH}_{2}\right), 116.8$ $(\mathrm{CN}), 120.8,122.4,126.7,128.3,133.6,134.3,138.5$, $145.9\left(\mathrm{C}_{6} \mathrm{H}_{4}\right.$, thiazole $\left.\mathrm{C}\right), 168.2,172.8(2 \times \mathrm{C}=\mathrm{N}) . \mathrm{MS}$ $(\mathrm{m} / \mathrm{z}) 255\left(\mathrm{M}^{+}, 25 \%\right)$.

\section{General procedure for the synthesis of the thiophene derivatives 11a-c}

To a solution of $5(1.99 \mathrm{~g}, 10 \mathrm{mmol})$ in dimethylformamide $(20 \mathrm{~mL})$ containing finely divided potassium hydroxide $(0.56 \mathrm{~g}, 10 \mathrm{mmol})$, phenylisothiocyanate $(1.35$ $\mathrm{g}, 10 \mathrm{mmol}$ ) was added. The reaction mixture was stirred at room temperature for $24 \mathrm{~h}$, then any of chloroacetone $(0.92 \mathrm{~g}, 10 \mathrm{mmol})$, ethyl chloroacetate $(1.22 \mathrm{~g}, 10 \mathrm{mmol})$ or $\alpha$-bromoacetophenone $(1.99 \mathrm{~g}, 10 \mathrm{mmol})$ was added. The whole reaction mixture was stirred at room temperature for additional $24 \mathrm{~h}$. The solid product formed upon dilution with ice/water mixture containing hydrochloric acid (till $\mathrm{pH} \mathrm{6)} \mathrm{was} \mathrm{collected} \mathrm{by} \mathrm{filtration} \mathrm{and} \mathrm{dried.} \mathrm{The}$ obtained product was crystallized from ethanol to give copper-coloured crystals for 11a and 11b and brown crystals for 11c.

\section{1-(4-(1-Acetyl-1H-benzo[d]imidazol-2-yl)-3-amino-5-} (phenylamino)thiophen-2-yl)ethanone (11a)

Yield $3.36 \mathrm{~g}(86 \%)$; m.p. $>300{ }^{\circ} \mathrm{C}$; Anal. Calcd. For $\mathrm{C}_{21} \mathrm{H}_{18} \mathrm{~N}_{4} \mathrm{O}_{2} \mathrm{~S}$ (390.46): C, 64.60; H, 4.65; N, 14.35; S, $8.21 \%$; found: C, $64.88 ; \mathrm{H}, 4.67 ; \mathrm{N}, 14.72 ; \mathrm{S}, 8.40 \%$. IR
(KBr) $v / \mathrm{cm}^{-1} 3456-3210(\mathrm{NH}), 3064(\mathrm{CH}$ aromatic), 2966-2875 (CH-alph.), $2192(\mathrm{CN}), 1730(\mathrm{C}=\mathrm{O}), 1598$, $1470(\mathrm{C}=\mathrm{C}), 1516(\mathrm{C}=\mathrm{N}) .{ }^{1} \mathrm{H}$ NMR $\left(\mathrm{DMSO}-d_{6}\right) \delta 1.84$, $1.89\left(2 \mathrm{~s}, 6 \mathrm{H}, 2 \times \mathrm{CH}_{3}\right), 5.48\left(\mathrm{~s}, 2 \mathrm{H}, \mathrm{NH}_{2}\right), 7.12-7.51$ $\left(\mathrm{m}, 9 \mathrm{H}, \mathrm{C}_{6} \mathrm{H}_{4}, \mathrm{C}_{6} \mathrm{H}_{5}\right), 12.71(\mathrm{~s}, 1 \mathrm{H}, \mathrm{NH}) .{ }^{13} \mathrm{C} \mathrm{NMR}$ $\left(\right.$ DMSO-d $\left.d_{6}\right) \delta 27.0\left(\mathrm{CH}_{3}\right), 38.8\left(\mathrm{CH}_{2}\right), 115.8(\mathrm{CN}), 121.4$, $123.1,124.9,125.1,126.2,127.9,128.2,129.4,130.3$, 133.2, 134.8, 136.8, 140.2, $142.1\left(\mathrm{C}_{6} \mathrm{H}_{4}, \mathrm{C}_{6} \mathrm{H}_{5}\right.$, thiophene C), 163.8, $166.2(2 \mathrm{C}=\mathrm{O}), 182.5(\mathrm{C}=\mathrm{N})$. MS $(\mathrm{m} / \mathrm{z}) 390$ $\left(\mathrm{M}^{+}, 38 \%\right)$.

Ethyl 4-(1-acetyl-1 $H$-benzo[ $d]$ imidazol-2-yl)-3-amino5-(phenylamino)-thiophene-2-carboxylate (11b)

Yield 2.94 g (70\%); m.p. $140{ }^{\circ} \mathrm{C}$; Anal. Calcd. for $\mathrm{C}_{22} \mathrm{H}_{20} \mathrm{~N}_{4} \mathrm{O}_{3} \mathrm{~S}$ (420.48): C, 62.84; H, 4.79; N, 13.32; S, 7.63\%; found: $\mathrm{C}, 62.73 ; \mathrm{H}, 4.52 ; \mathrm{N}, 13.06 ; \mathrm{S}, 7.67 \%$. IR (KBr) $v / \mathrm{cm}^{-1} 3441-3211(\mathrm{NH}), 3063(\mathrm{CH}$ aromatic), 2970-2876 (CH-alph.), $2193(\mathrm{CN}), 1725(\mathrm{C}=\mathrm{O}), 1597$, $1472(\mathrm{C}=\mathrm{C}), 1516(\mathrm{C}=\mathrm{N}) .{ }^{1} \mathrm{H}$ NMR $\left(\mathrm{DMSO}-d_{6}\right) \delta$ 1.16-1.21 (t, 3H, $\left.\mathrm{CH}_{3}\right), 2.11\left(\mathrm{~s}, 3 \mathrm{H}, \mathrm{CH}_{3}\right), 3.97(\mathrm{~s}, 2 \mathrm{H}$, $\left.\mathrm{NH}_{2}\right), 4.11-4.16\left(\mathrm{q}, 2 \mathrm{H}, \mathrm{CH}_{2}\right), 6.76-7.55\left(\mathrm{~m}, 9 \mathrm{H}, \mathrm{C}_{6} \mathrm{H}_{4}\right.$ $\mathrm{C}_{6} \mathrm{H}_{5}$ ), $12.74(\mathrm{~s}, 1 \mathrm{H}, \mathrm{NH}) .{ }^{13} \mathrm{C}$ NMR (DMSO- $\left.d_{6}\right) \delta 16.3$ (ester $\left.\mathrm{CH}_{3}\right), 28.2\left(\mathrm{CO}-\mathrm{CH}_{3}\right), 52.8\left(\right.$ ester $\left.\mathrm{CH}_{2}\right), 115.9$ (CN), 120.6, 122.8, 123.4, 125.0, 125.8, 126.3, 126.8, $128.1,130.3,133.4,136.1,138.9,141.8,142.0\left(\mathrm{C}_{6} \mathrm{H}_{4}\right.$, $\mathrm{C}_{6} \mathrm{H}_{5}$, thiophene $\left.\mathrm{C}\right), 164.3,166.9(2 \times \mathrm{C}=\mathrm{O}), 172.8(\mathrm{C}=\mathrm{N})$. MS $(\mathrm{m} / \mathrm{z}) 420\left(\mathrm{M}^{+}, 26 \%\right)$.

1-(2-(4-Amino-5-benzoyl-2-(phenylamino)thiophen-3yl)-1H-benzo[d]imidazol-1-yl)ethanone (11c)

Yield $2.26 \mathrm{~g}(50 \%)$; m.p. $>300{ }^{\circ} \mathrm{C}$; Anal. Calcd. for $\mathrm{C}_{26} \mathrm{H}_{20} \mathrm{~N}_{4} \mathrm{O}_{2} \mathrm{~S}$ (452.53): C, 69.01; H, 4.45; N, 12.38; S, 7.09\%; found: C, 68.89; H, 4.65; N, 12.09; S, 6.83\%. IR $(\mathrm{KBr}) \mathrm{v} / \mathrm{cm}^{-1} 3431-3212\left(\mathrm{NH}, \mathrm{NH}_{2}\right), 3067(\mathrm{CH}$ aromatic), 2968-2879 (CH-alph.), 2195 (CN), 1690, 1627 (2 $\mathrm{C}=\mathrm{O}), 1599,1471(\mathrm{C}=\mathrm{C}), 1516(\mathrm{C}=\mathrm{N}) .{ }^{1} \mathrm{H}$ NMR (DMSO$\left.d_{6}\right) \delta 2.12\left(\mathrm{~s}, 3 \mathrm{H}, \mathrm{CH}_{3}\right), 2.73\left(\mathrm{~s}, 2 \mathrm{H}, \mathrm{D}_{2} \mathrm{O}\right.$ exchangeable, $\left.\mathrm{NH}_{2}\right), 7.21-8.09\left(\mathrm{~m}, 14 \mathrm{H}, \mathrm{C}_{6} \mathrm{H}_{4}, 2 \times \mathrm{C}_{6} \mathrm{H}_{5}\right), 8.74(\mathrm{~s}, 1 \mathrm{H}$, $\mathrm{D}_{2} \mathrm{O}$ exchangeable, $\mathrm{NH}$ ). ${ }^{13} \mathrm{C}$ NMR (DMSO- $\left.d_{6}\right) \delta 28.6$ $\left(\mathrm{CO}-\mathrm{CH}_{3}\right), 119.3,121.9,124.2,125.8,125.8,126.0$, $126.8,127.1,127.9,128.1,130.3,133.4,136.1,138.9$, 140.3, 141.2, 142.6, $143.8\left(2 \times \mathrm{C}_{6} \mathrm{H}_{5}\right.$, thiophene $\left.\mathrm{C}\right), 163.9$, $165.2(2 \times \mathrm{C}=\mathrm{O}), 170.6(\mathrm{C}=\mathrm{N})$. MS $(\mathrm{m} / \mathrm{z}) 452\left(\mathrm{M}^{+}, 42 \%\right)$.

\section{General procedure for the synthesis of the benzylidene derivatives 12a,b}

To a solution of $5(1.99 \mathrm{~g}, 10 \mathrm{mmol})$ in dimethylformamide $(25 \mathrm{~mL})$ containing piperidine $(0.5 \mathrm{~mL})$, either of benzaldehyde ( $1.06 \mathrm{~g}, 10 \mathrm{mmol})$ or salicylaldehyde (1.22 $\mathrm{g}, 10 \mathrm{mmol}$ ) was added. The reaction mixture was heated under reflux for $3 \mathrm{~h}$ then poured into a beaker containing ice/water mixture containing a few drops of hydrochloric acid. The solid product formed was collected by filtration and dried. The obtained product was crystallized from ethanol to give yellow crystals of 12a and orange crystals of $12 b$. 


\section{2-(1-Acetyl-1H-benzo[d]imidazol-2-yl)-3-phenylacry- lonitrile (12a)}

Yield $1.81 \mathrm{~g}$ (63\%); m.p. $>300{ }^{\circ} \mathrm{C}$; Anal. Calcd. for $\mathrm{C}_{18} \mathrm{H}_{13} \mathrm{~N}_{3} \mathrm{O}$ (287.32): $\mathrm{C}, 75.25 ; \mathrm{H}, 4.56 ; \mathrm{N}, 14.63 \%$; found: C, $74.33 ; \mathrm{H}, 4.36 ; \mathrm{N}, 14.49 \%$. IR (KBr) $v / \mathrm{cm}^{-1}$ 3066 (CH aromatic), 2967-2876 (CH-alph.), $2193(\mathrm{CN})$, $1627(\mathrm{C}=\mathrm{O}), 1599,1470(\mathrm{C}=\mathrm{C}), 1515(\mathrm{C}=\mathrm{N}) .{ }^{1} \mathrm{H}$ NMR $\left(\mathrm{DMSO}-d_{6}\right) \delta 2.00\left(\mathrm{~s}, 3 \mathrm{H}, \mathrm{CH}_{3}\right), 3.30(\mathrm{~s}, 1 \mathrm{H}, \mathrm{CH})$, 7.21-7.51 (m, 9H, $\left.\mathrm{C}_{6} \mathrm{H}_{4} \mathrm{C}_{6} \mathrm{H}_{5}\right) .{ }^{13} \mathrm{C}$ NMR (DMSO- $\left.d_{6}\right) \delta$ $28.4\left(\mathrm{CO}-\mathrm{CH}_{3}\right), 88.5,90.6(\mathrm{CH}=\mathrm{C}), 121.6,123.8,124.2$, $124.9, \quad 125 . \overline{0}, 127.1,130.3,133.4,138.9,139.0$ $\left(2 \times \mathrm{C}_{6} \mathrm{H}_{5}\right), 165.8(\mathrm{C}=\mathrm{O}), 171.2(\mathrm{C}=\mathrm{N}) . \mathrm{MS}(\mathrm{m} / \mathrm{z}) 287$ $\left(\mathrm{M}^{+}, 23 \%\right)$.

\section{2-(1-Acetyl-1 $H$-benzo[d]imidazol-2-yl)-3-(2-hydroxyp- henyl)acrylonitrile (12b)}

Yield 2.79 g (92\%); m.p. $>300{ }^{\circ} \mathrm{C}$; Anal. Calcd. for $\mathrm{C}_{18} \mathrm{H}_{13} \mathrm{~N}_{3} \mathrm{O}_{2}$ (303.31): C, 71.28; $\mathrm{H}, 4.32 ; \mathrm{N}, 13.85 \%$; found: C, 71.33; H, 4.88; N, 9.04\%. IR (KBr) v/cm 3432-3215 $(\mathrm{OH}), 3100(\mathrm{CH}$ aromatic $), 2999-2882$ (CH-alph.), $2194(\mathrm{CN}), 1633(\mathrm{C}=\mathrm{O}), 1601,1477(\mathrm{C}=\mathrm{C})$, $1518(\mathrm{C}=\mathrm{N}) .{ }^{1} \mathrm{H}$ NMR $\left(\mathrm{DMSO}-d_{6}\right) \delta 1.20\left(\mathrm{~s}, 3 \mathrm{H}, \mathrm{CH}_{3}\right)$, $3.57(\mathrm{~s}, 1 \mathrm{H}, \mathrm{CH}), 7.21-7.51\left(\mathrm{~m}, 8 \mathrm{H}, 2 \mathrm{C}_{6} \mathrm{H}_{4}\right), 12.75(\mathrm{~s}$, $1 \mathrm{H}, \mathrm{D}_{2} \mathrm{O}$ exchangeable, OH). ${ }^{13} \mathrm{C}$ NMR (DMSO- $\left.d_{6}\right) \delta$ $28.6\left(\mathrm{CO}-\mathrm{CH}_{3}\right), 88.9,90.8(\mathrm{CH}=\mathrm{C}), 120.8,122.5,124.2$, $124.6,125 . \overline{6}, 126.8,128.7,133.4,136.3,140.3$ $\left(2 \times \mathrm{C}_{6} \mathrm{H}_{5}\right), 164.6(\mathrm{C}=\mathrm{O}), 170.8(\mathrm{C}=\mathrm{N}) . \mathrm{MS}(\mathrm{m} / \mathrm{z}) 303$ $\left(\mathrm{M}^{+}, 36 \%\right)$.

\section{General procedure for the synthesis of the ben- zo[4,5]imidazo[1,2-a]pyridine derivatives 13a,b}

To a solution of $\mathbf{5}(1.99 \mathrm{~g}, 10 \mathrm{mmol})$ in dimethylformamide $(30 \mathrm{~mL})$ containing triethylamine $(0.5 \mathrm{~mL})$, either malononitrile $(0.66 \mathrm{~g}, 10 \mathrm{mmol})$ or ethyl cyanoacetate $(1.13 \mathrm{~g}, 10 \mathrm{mmol})$ was added. The reaction mixture was heated under reflux for $4 \mathrm{~h}$ then poured into a beaker containing ice/water mixture containing a few drops of hydrochloric acid. The solid product formed was collected by filtration and dried. The obtained product was crystallized from ethanol to give buff crystals of 13a and yellowish brown crystals of $\mathbf{1 3 b}$.

\section{3-Amino-1-methylbenzo[4,5]imidazo[1,2-a]pyridine- 2,4-dicarbonitrile (13a)}

Yield $2.04 \mathrm{~g}(82 \%)$; m.p. $>300{ }^{\circ} \mathrm{C}$; Anal. Calcd. For $\mathrm{C}_{14} \mathrm{H}_{11} \mathrm{~N}_{5}$ (249.27): C, 67.46; H, 4.45; N, 28.10\%; found: C, 67.06; H, 4.18; N, 28.43\%. IR (KBr) v/cm 3470-3212 (NH, NH $), 3077$ (CH aromatic), 2971-2877 (CH-alph.), 2223, 2194 (2CN), 1599, $1474(\mathrm{C}=\mathrm{C}), 1517$ $(\mathrm{C}=\mathrm{N}) .{ }^{1} \mathrm{H}$ NMR $\left(\mathrm{DMSO}-d_{6}\right) \delta 1.16\left(\mathrm{~s}, 3 \mathrm{H}, \mathrm{CH}_{3}\right), 5.51(\mathrm{~s}$, $2 \mathrm{H}, \mathrm{D}_{2} \mathrm{O}$ exchangeable, $\left.\mathrm{NH}_{2}\right), 6.30\left(\mathrm{~s}, 1 \mathrm{H}\right.$, pyridine $\left.\mathrm{C}_{6}\right)$, 7.21-7.50 (m, 4H, $\left.\mathrm{C}_{6} \mathrm{H}_{4}\right), 12.75(\mathrm{~s}, 1 \mathrm{H}, \mathrm{NH}) .{ }^{13} \mathrm{C} \mathrm{NMR}$ $\left(\right.$ DMSO- $\left.d_{6}\right) \delta 22.7\left(\mathrm{CH}_{3}\right), 116.8,117.3(2 \times \mathrm{CN}), 119.8$, $120.7,122.5,124.8,125.3,126.4,128.0,130.9,133.2$, $134.8,143.2\left(\mathrm{C}_{6} \mathrm{H}_{4}\right.$, imidazole, pyridine $\left.\mathrm{C}\right), 173.8(\mathrm{C}=\mathrm{N})$. MS $(\mathrm{m} / \mathrm{z}) 249\left(\mathbf{M}^{+}, 22 \%\right)$.
Ethyl 3-amino-4-cyano-1-methylbenzo[4,5]imidazo [1,2-a]pyridine-2-carboxylate (13b)

Yield $2.01 \mathrm{~g}(68 \%) ;$ m.p. $>300{ }^{\circ} \mathrm{C}$; Anal. Calcd. for $\mathrm{C}_{16} \mathrm{H}_{16} \mathrm{~N}_{4} \mathrm{O}_{2}$ (296.32): C, 64.85; H, 5.44; N, 18.91\%; found: C, 64.92; H, 4.27; N, $18.73 \%$. IR (KBr) v/ $\mathrm{cm}^{-1}$ 3426-3214 (NH, NH $), 3103(\mathrm{CH}$ aromatic), $2883(\mathrm{CH}-$ alph.), $2194(\mathrm{CN}), 1750(\mathrm{C}=\mathrm{O}$, ester), 1601, $1472(\mathrm{C}=\mathrm{C})$. ${ }^{1} \mathrm{H}$ NMR (DMSO- $\left.d_{6}\right) \delta 1.10\left(\mathrm{t}, 3 \mathrm{H}, \mathrm{CH}_{3}\right), 2.12(\mathrm{~s}, 3 \mathrm{H}$, $\mathrm{CH}_{3}$ ), 3.29 (q, 2H, $\mathrm{CH}_{2}$ ), 5.01 (s, $2 \mathrm{H}, \mathrm{D}_{2} \mathrm{O}$ exchangeable, $\left.\mathrm{NH}_{2}\right), 6.10\left(\mathrm{~s}, 1 \mathrm{H}\right.$, pyridine $\left.\mathrm{C}_{6}\right), 7.20-7.51\left(\mathrm{~m}, 4 \mathrm{H}, \mathrm{C}_{6} \mathrm{H}_{4}\right)$, $12.73\left(\mathrm{~s}, 1 \mathrm{H}, \mathrm{D}_{2} \mathrm{O}\right.$ exchangeable, $\left.\mathrm{NH}\right) .{ }^{13} \mathrm{C} \mathrm{NMR}$ $\left(\right.$ DMSO- $\left.d_{6}\right) \delta 16.8$ (ester $\left.\mathrm{CH}_{3}\right), 22.4\left(\mathrm{CH}_{3}\right), 56.9$ (ester $\left.\mathrm{CH}_{2}\right), 116.5,117.1(2 \times \mathrm{CN}), 119.9,121.3,122.8,124.4$, $125.1,126.8,127.4,130.3,133.6,134.1,143.8\left(\mathrm{C}_{6} \mathrm{H}_{4}\right.$, imidazole, pyridine $\mathrm{C}), 162.8(\mathrm{C}=\mathrm{O})$. MS $(\mathrm{m} / \mathrm{z}) 296\left(\mathrm{M}^{+}, 35 \%\right)$.

\section{2-(1-Acetyl-1 $H$-benzo[d]imidazol-2-yl)-3-phenylbut-2- enenitrile (14)}

To a mixture of $5(1.99 \mathrm{~g}, 10 \mathrm{mmol})$ and acetophenone $(1.35 \mathrm{~g}, 10 \mathrm{mmol})$, ammonium acetate $(0.77 \mathrm{~g}, 10$ mmol) was added. The reaction mixture was heated in oil bath at $140{ }^{\circ} \mathrm{C}$ for $1 \mathrm{~h}$ then left to cool. The semisolid formed was triturated with ethanol $(40 \mathrm{~mL})$ and the formed solid product was collected by filtration and dried. The obtained product was crystallized from ethanol to give dark brown crystals.

Yield 1.99 g (66\%); m.p. $210-213{ }^{\circ} \mathrm{C}$; Anal. Calcd. for $\mathrm{C}_{19} \mathrm{H}_{15} \mathrm{~N}_{3} \mathrm{O}$ (301.34): C, 75.73; H, 5.02; N, 13.94\%; found: C, 74.83; H, 5.39; N, $13.85 \%$. IR (KBr) v/ $\mathrm{cm}^{-1}$ 3086 (CH aromatic), 2880 (CH-alph.), $2194(\mathrm{CN}), 1630$ $(\mathrm{C}=\mathrm{O}), 1599,1472(\mathrm{C}=\mathrm{C}), 1517(\mathrm{C}=\mathrm{N}) .{ }^{1} \mathrm{H}$ NMR $\left(\mathrm{DMSO}-d_{6}\right) \delta 1.20,2.40\left(2 \mathrm{~s}, 6 \mathrm{H}, 2 \times \mathrm{CH}_{3}\right), 7.21-7.52(\mathrm{~m}$, $\left.9 \mathrm{H}, \mathrm{C}_{6} \mathrm{H}_{4}, \mathrm{C}_{6} \mathrm{H}_{5}\right) \cdot{ }^{13} \mathrm{C}$ NMR (DMSO- $\left.d_{6}\right) \delta 22.4,28.6\left(\mathrm{CH}_{3}\right.$, $\left.\mathrm{CO}-\mathrm{CH}_{3}\right), 88.9,90.6(\mathrm{CH}=\mathrm{C}), 121.3,122.5,124.2,124.6$, $126.1,126.8,127.2,133.4,136.3,136.9\left(2 \times \mathrm{C}_{6} \mathrm{H}_{5}\right), 164.6$ $(\mathrm{C}=\mathrm{O}), 170.6(\mathrm{C}=\mathrm{N}) . \mathrm{MS}(\mathrm{m} / \mathrm{z}) 301\left(\mathrm{M}^{+}, 17 \%\right)$.

\section{2-(1-Acetyl-1H-benzo[d]imidazol-2-yl)-3,5-diphenyl- penta-2,4-dienenitrile (15)}

To a solution of $14(3.01 \mathrm{~g}, 10 \mathrm{mmol})$ in dimethylformamide $(25 \mathrm{~mL})$ containing piperidine $(0.50 \mathrm{~mL})$, benzaldehyde $(1.06 \mathrm{~g}, 10 \mathrm{mmol})$ was added. The reaction mixture was heated under reflux for $4 \mathrm{~h}$ then poured into a beaker containing ice/water mixture containing a few drops of hydrochloric acid. The solid product formed was collected by filtration and dried. The obtained product was crystallized from ethanol to give brown crystals.

Yield $2.57 \mathrm{~g}(66 \%)$; m.p. $150{ }^{\circ} \mathrm{C}$; Anal. Calcd. for $\mathrm{C}_{26} \mathrm{H}_{19} \mathrm{~N}_{3} \mathrm{O}$ (389.45): C, 80.18; H, 4.92; N, 10.79\%; found: C, 80.29; H, 5.14; N, 10.59\%. IR (KBr) v/ $\mathrm{cm}^{-1}$ 3057 (CH aromatic), 2927 (CH-alph.), $2195(\mathrm{CN}), 1627$ $(\mathrm{C}=\mathrm{O}), 1596(\mathrm{C}=\mathrm{N}), 1489(\mathrm{C}=\mathrm{C}) .{ }^{1} \mathrm{H}$ NMR $\left(\mathrm{DMSO}-d_{6}\right) \delta$ 2.23 (s, 3H, $\left.\mathrm{CH}_{3}\right), 2.72,2.89(2 \mathrm{~s}, 2 \mathrm{H}, 2 \times \mathrm{CH}), 6.50-8.35$ $\left(\mathrm{m}, 14 \mathrm{H}, \mathrm{C}_{6} \mathrm{H}_{4}, 2 \times \mathrm{C}_{6} \mathrm{H}_{5}\right.$ ). ${ }^{13} \mathrm{C}$ NMR (DMSO- $\left.d_{6}\right) \delta 24.3$ $\left(\mathrm{CH}_{3}\right), 98.5,104.8(\mathrm{C}=\mathrm{C}), 116.4(\mathrm{CN}), 120.4,121.3$, $122.4,124.9,125.8,126.8,127.8,128.0,130.6,133.5$, 
136.5, $140.8\left(\mathrm{C}_{6} \mathrm{H}_{4}, 2 \times \mathrm{C}_{6} \mathrm{H}_{5}\right), 164.3(\mathrm{C}=\mathrm{O}), 173.1(\mathrm{C}=\mathrm{N})$. MS $(\mathrm{m} / \mathrm{z}) 389\left(\mathbf{M}^{+}, 44 \%\right)$.

General procedure for the synthesis of the thiophene derivatives 16a,b

To a solution of $5(1.99 \mathrm{~g}, 10 \mathrm{mmol})$ in 1,4-dioxane $(25 \mathrm{~mL})$ containing triethylamine $(0.50 \mathrm{~mL})$ and elemental sulfur $(0.32 \mathrm{~g}, 10 \mathrm{mmol})$ either malononitrile $(0.66 \mathrm{~g}$, $10 \mathrm{mmol})$ or ethyl cyanoacetate $(1.13 \mathrm{~g}, 10 \mathrm{mmol})$ was added. The reaction mixture was heated under reflux for $4 \mathrm{~h}$ then poured into a beaker containing ice/water mixture containing a few drops of hydrochloric acid. The solid product was collected by filtration and dried. The obtained product was crystallized from ethanol to give dark brown crystals of $\mathbf{1 6 a}$ and light brown crystals of $\mathbf{1 6} \mathbf{b}$.

5-(1-Acetyl-1H-benzo[d]imidazol-2-yl)-2,4-diaminothiophene-3-carbonitrile (16a) Yield $2.08 \mathrm{~g}$ (70\%); m.p. 288-293 ${ }^{\circ} \mathrm{C}$; Anal. Calcd. for $\mathrm{C}_{14} \mathrm{H}_{11} \mathrm{~N}_{5} \mathrm{OS}$ (297.34): C, $56.55 ; \mathrm{H}, 3.73 ; \mathrm{N}, 23.55 ; \mathrm{S}, 10.78 \%$; found: $\mathrm{C}, 56.43 ; \mathrm{H}$, 3.49; N, 23.70; S, 10.63\%. IR (KBr) v/cm ${ }^{-1} 3426-3213$ $\left(2 \times \mathrm{NH}_{2}\right), 3050(\mathrm{CH}$ aromatic), 2973, $2882(\mathrm{CH}-$ alph.), $2195(\mathrm{CN}), 1680(\mathrm{C}=\mathrm{O}), 1600(\mathrm{C}=\mathrm{N}), 1470(\mathrm{C}=\mathrm{C}), 1518$ $(\mathrm{C}=\mathrm{N}) .{ }^{1} \mathrm{H}$ NMR $\left(\right.$ DMSO- $\left.d_{6}\right) \delta 2.06\left(\mathrm{~s}, 3 \mathrm{H}, \mathrm{CH}_{3}\right), 2.73$, $2.89\left(2 \mathrm{~s}, 4 \mathrm{H}, \mathrm{D}_{2} \mathrm{O}\right.$ exchangeable, $\left.2 \times \mathrm{NH}_{2}\right), 7.20-7.95(\mathrm{~m}$, $\left.4 \mathrm{H}, \mathrm{C}_{6} \mathrm{H}_{4}\right) \cdot{ }^{13} \mathrm{C}$ NMR (DMSO- $\left.d_{6}\right) \delta 22.6\left(\mathrm{CH}_{3}\right), 116.3$ (CN), 119.8, 120.7, 122.6, 124.6, 129.5, 130.4, 133.5, 142.8, 143.2, $144.3\left(\mathrm{C}_{6} \mathrm{H}_{4}\right.$, thiophene $\left.\mathrm{C}\right), 163.2(\mathrm{C}=\mathrm{O})$, $172.3(\mathrm{C}=\mathrm{N})$. MS $(\mathrm{m} / \mathrm{z}) 297\left(\mathrm{M}^{+}, 32 \%\right)$.

Ethyl 5-(1-acetyl-1 $H$-benzo[d]imidazol-2-yl)-2,4-diaminothiophene-3-carboxylate (16b)

Yield $2.38 \mathrm{~g}(69 \%)$; m.p. $>300{ }^{\circ} \mathrm{C}$; Anal. Calcd. for $\mathrm{C}_{16} \mathrm{H}_{16} \mathrm{~N}_{4} \mathrm{O}_{3} \mathrm{~S}$ (344.39): $\mathrm{C}, 55.80 ; \mathrm{H}, 4.68 ; \mathrm{N}, 16.27 ; \mathrm{S}$, 9.31\%; found: C, 55.89, H, 4.29; N, 16.60; S, 8.04\%. IR (KBr) $v / \mathrm{cm}^{-1}$ 3430-3216 (2 $\left.\mathrm{NH}_{2}\right), 3107$ (CH aromatic), 2978, 2884 (CH-alph.), 1633 (C=O), 1750 (C=O, ester), $1601(\mathrm{C}=\mathrm{N}), 1471(\mathrm{C}=\mathrm{C}), 1518(\mathrm{C}=\mathrm{N}) .{ }^{1} \mathrm{H}$ NMR $\left(\right.$ DMSO- $\left.d_{6}\right) \delta 1.04\left(\mathrm{t}, 3 \mathrm{H}, \mathrm{CH}_{3}\right), 2.33\left(\mathrm{~s}, 3 \mathrm{H}, \mathrm{CH}_{3}\right), 4.28$ $\left(\mathrm{q}, 2 \mathrm{H}, \mathrm{CH}_{2}\right), 5.80,5.95\left(2 \mathrm{~s}, 4 \mathrm{H}, \mathrm{D}_{2} \mathrm{O}\right.$ exchangeable, 2 $\left.\mathrm{NH}_{2}\right), 7.21-7.51\left(\mathrm{~m}, 4 \mathrm{H}, \mathrm{C}_{6} \mathrm{H}_{4}\right) .{ }^{13} \mathrm{C}$ NMR $\left(\right.$ DMSO- $\left.d_{6}\right) \delta$ 16.2 (ester $\left.\mathrm{CH}_{3}\right), 22.8\left(\mathrm{CH}_{3}\right), 120.3,120.8,121.2,123.8$, 124.8, 125.8, 134.8, 142.8, 143.6, $144.9\left(\mathrm{C}_{6} \mathrm{H}_{4}\right.$, thiophene C), $164.8(\mathrm{C}=\mathrm{O}), 172.0(\mathrm{C}=\mathrm{N})$. $\mathrm{MS}(\mathrm{m} / \mathrm{z}) 344\left(\mathrm{M}^{+}, 48 \%\right)$.

\section{2-(2-Phenylhydrazono)-2-(1-acetyl-1H-benzo $[d]$ imida-} zol-2-yl) acetonitrile (17)

To a cold solution $\left(0-5{ }^{\circ} \mathrm{C}\right)$ of $5(1.99 \mathrm{~g}, 10 \mathrm{mmol})$ in ethanol $(50 \mathrm{~mL})$ containing sodium hydroxide solution $(10 \mathrm{~mL}, 10 \%)$ and a solution of benzenediazonium chloride $(10 \mathrm{mmol})$ [which was prepared by dissolving sodium nitrite $(0.70 \mathrm{~g}, 10 \mathrm{mmol})$ in water, $2 \mathrm{~mL}$ was added to a cold solution of aniline $(0.93 \mathrm{~g}, 10 \mathrm{mmol})$ containing appropriate amount of hydrochloric acid and with continuous stirring] was added with continuous stirring. The solid product formed was collected by filtration and dried. The obtained product was crystallized from ethanol to give brown crystals.

Yield $2.24 \mathrm{~g}$ (74\%); m.p. $265{ }^{\circ} \mathrm{C}$; Anal. Calcd for $\mathrm{C}_{17} \mathrm{H}_{13} \mathrm{~N}_{5} \mathrm{O}$ (303.32): C, 67.32; H, 4.32; N, 23.09\%; found: C, 67.29; H, 4.09; N, 23.27\%. IR (KBr) v/cm ${ }^{-1}$ 3423-3214 (NH), 3098 (CH aromatic), 2976-2883 (CHalph.), $2194(\mathrm{CN}), 1631(\mathrm{C}=\mathrm{O}), 1521$ (=N-NH), 1601, $1475(\mathrm{C}=\mathrm{C}) .{ }^{1} \mathrm{H}$ NMR (DMSO- $\left.d_{6}\right) \delta 2.21\left(\mathrm{~s}, 3 \mathrm{H}, \mathrm{CH}_{3}\right)$, 7.21-7.51 (m, 9H, $\left.\mathrm{C}_{6} \mathrm{H}_{4}, \mathrm{C}_{6} \mathrm{H}_{5}\right), 12.76\left(\mathrm{~s}, 1 \mathrm{H}, \mathrm{D}_{2} \mathrm{O}\right.$ exchangeable, NH). ${ }^{13} \mathrm{C}$ NMR (DMSO- $\left.d_{6}\right) \delta 22.8\left(\mathrm{CH}_{3}\right)$, $115.9(\mathrm{CN}), 120.4,120.7,123.2,124.6,126.5,128.6$, 133.5, 142.8, 143.2, $144.3\left(\mathrm{C}_{6} \mathrm{H}_{5}, \mathrm{C}_{6} \mathrm{H}_{4}\right), 164.0(\mathrm{C}=\mathrm{O})$, $172.8(\mathrm{C}=\mathrm{N})$. MS $(\mathrm{m} / \mathrm{z}) 303\left(\mathrm{M}^{+}, 20 \%\right)$.

General procedure for the synthesis of the phenylhydrazone derivatives $18 \mathrm{a}, \mathrm{b}$

To a solution of $\mathbf{1 7}(3.03 \mathrm{~g}, 10 \mathrm{mmol})$ in 1,4-dioxane $(30 \mathrm{~mL})$ containing triethylamine $(0.50 \mathrm{~mL})$ and elemental sulfur $(0.32 \mathrm{~g}, 10 \mathrm{mmol})$ either malononitrile $(0.66 \mathrm{~g}$, $10 \mathrm{mmol})$ or ethyl cyanoacetae $(1.13 \mathrm{~g}, 10 \mathrm{mmol})$ was added. The reaction mixture was heated under reflux for $4 \mathrm{~h}$ then poured into a beaker containing ice/water mixture containing a few drops of hydrochloric acid. The solid product was collected by filtration and dried. The obtained product was crystallized from ethanol to give yellow crystals of 18a and light green crystals of $\mathbf{1 8 b}$.

4-(2-((2-Phenylhydrazono)(cyano)methyl)- $1 H$-benzo [d]imidazol-1-yl)-2-aminothiophene-3-carbonitrile (18a)

Yield 3.45 g (90\%); m.p. $198{ }^{\circ} \mathrm{C}$; Anal. Calcd. for $\mathrm{C}_{20} \mathrm{H}_{13} \mathrm{~N}_{7} \mathrm{~S}$ (383.43): C, 62.65; H, 3.42; N, 25.57; S, 8.36\%; found: C, 62.88; H, 3.59; N, 25.19; S, 8.07\%. IR (KBr) $v / \mathrm{cm}^{-1} 3352-3209\left(\mathrm{NH}, \mathrm{NH}_{2}\right), 3100(\mathrm{CH}$-aromatic), 2922 (CH-alph.), $2196(\mathrm{CN}), 1547(\mathrm{C}=\mathrm{N}), 1463$ $(\mathrm{C}=\mathrm{C}) .{ }^{1} \mathrm{H}$ NMR (DMSO- $\left.d_{6}\right) \delta 5.60\left(\mathrm{~s}, 2 \mathrm{H}, \mathrm{D}_{2} \mathrm{O}\right.$ exchangeable, $\left.\mathrm{NH}_{2}\right), 7.20\left(\mathrm{~s}, 1 \mathrm{H}\right.$, thiophene $\left.\mathrm{C}_{5}\right), 7.21-7.67(\mathrm{~m}$, $9 \mathrm{H}, \mathrm{C}_{6} \mathrm{H}_{4} \mathrm{C}_{6} \mathrm{H}_{5}$ ), 12.75 (s, 1H, NH). ${ }^{13} \mathrm{C}$ NMR (DMSO$\left.d_{6}\right) \delta 116.3$. $116.8(2 \times \mathrm{CN}), 120.0,120.6,123.2,125.1$, 126.5, 127.2, 136.5, 138.1, 139.2, 140.6, 140.8, 141.3, $142.6\left(\mathrm{C}_{6} \mathrm{H}_{5}, \mathrm{C}_{6} \mathrm{H}_{4}\right.$, thiophene $\left.\mathrm{C}\right), 164.0(\mathrm{C}=\mathrm{O}), 172.8$ $(\mathrm{C}=\mathrm{N})$. MS $(\mathrm{m} / \mathrm{z}) 383\left(\mathrm{M}^{+}, 18 \%\right)$.

Ethyl 4-(2-((2-phenylhydrazono)(cyano)methyl)-1Hbenzo $[d]$ imidazol-1-yl)-2-aminothiophene-3-carboxylate (18b)

Yield $2.54 \mathrm{~g}(59 \%)$; m.p. $>300{ }^{\circ} \mathrm{C}$; Anal. Calcd. for $\mathrm{C}_{22} \mathrm{H}_{18} \mathrm{~N}_{6} \mathrm{O}_{2} \mathrm{~S}$ (430.48): $\mathrm{C}, 61.38 ; \mathrm{H}, 4.21 ; \mathrm{N}, 19.52 ; \mathrm{S}$, 7.45\%; found: C, 61.03; H, 4.58; N, 19.71; S, 7.66\%. IR $(\mathrm{KBr}) \mathrm{v} / \mathrm{cm}^{-1} 3427-3215\left(\mathrm{NH}, \mathrm{NH}_{2}\right), 3100(\mathrm{CH}$ aromatic), 2900 (CH-alph.), $2194(\mathrm{CN}), 1632(\mathrm{C}=\mathrm{O}), 1520$ $(\mathrm{C}=\mathrm{N}), 1601,1475(\mathrm{C}=\mathrm{C}) .{ }^{1} \mathrm{H}$ NMR $\left(\mathrm{DMSO}-d_{6}\right) \delta 1.10(\mathrm{t}$, $\left.3 \mathrm{H}, \mathrm{CH}_{3}\right), 4.21\left(\mathrm{q}, 2 \mathrm{H}, \mathrm{CH}_{2}\right), 5.01\left(\mathrm{~s}, 2 \mathrm{H}, \mathrm{D}_{2} \mathrm{O}\right.$ exchangeable, $\left.\mathrm{NH}_{2}\right), 7.20\left(\mathrm{~s}, 1 \mathrm{H}\right.$, thiophene $\left.\mathrm{C}_{5}\right), 7.21-7.51(\mathrm{~m}$, $\left.9 \mathrm{H}, \mathrm{C}_{6} \mathrm{H}_{4}, \mathrm{C}_{6} \mathrm{H}_{5}\right), 12.77$ (s,1H, $\mathrm{D}_{2} \mathrm{O}$ exchangeable, $\left.\mathrm{NH}\right)$. ${ }^{13} \mathrm{C}$ NMR (DMSO- $d_{6}$ ) $\delta 16.3\left(\right.$ ester $\mathrm{CH}_{3}$ ), 54.8 (ester 
$\left.\mathrm{CH}_{2}\right), 116.5(\mathrm{CN}), 120.3,121.8,123.8,125.1,127.2$, 128.6, 130.2, 138.1, 139.2, 139.9, 140.4, 142.9, 143.8 $\left(\mathrm{C}_{6} \mathrm{H}_{5}, \mathrm{C}_{6} \mathrm{H}_{4}\right.$, thiophene $\left.\mathrm{C}\right), 164.0(\mathrm{C}=\mathrm{O}), 172.8(\mathrm{C}=\mathrm{N})$. $\operatorname{MS}(\mathrm{m} / z) 430\left(\mathrm{M}^{+}, 22 \%\right)$.

\section{Conclusions}

In the present study we have synthesized a series of heterocyclic derivatives of 2-cyanomethylbenzo[c]imidazole 1 . The newly synthesized products were tested against MCF-7 (breast adenocarcinoma), NCI-H460 (non-small cell lung cancer), and SF-268 (CNS cancer) and the results showed that compounds $2 \mathrm{~b}, \mathbf{6}, \mathbf{1 1 b}, \mathbf{1 1 c}, \mathbf{1 2 b}, \mathbf{1 6 a}, \mathbf{1 6 b}$ and 18a exhibited optimal cytotoxic effect against cancer cell lines. The toxicity of these optimal cytotoxic compounds was monitored via in vivo lethality to shrimp larvae (Artemia salina). The results showed that compounds $\mathbf{1 1 b} \mathbf{1 2 b}$, and $\mathbf{1 6 b}$ are non toxic towards shrimp larvae.

\section{References}

1. H. Irannejad, M. Amini, F. Khodagholi, N. Ansari, S. K. Tusi, M. Sharifzadeh, A. Shafiee, Bioorg. Med. Chem. 2010, 18, 4224-4230.

http://dx.doi.org/10.1016/j.bmc.2010.04.097

2. N. Catozzi, M. G. Edwards, S. A. Raw, P. Wasnaire, R. K. Taylor, J. Org. Chem. 2009, 74, 8343-8354. http://dx.doi.org/10.1021/jo901761r

3. S. Laphookhieo, S. Jones, S. A. Raw, Y. F. Sainz, R. J. K. Taylor, Tetrahedron Lett. 2006, 47, 3865-3870. http://dx.doi.org/10.1016/j.tetlet.2006.03.178

4. D. L. Boger, J. S. Panek, M. Yasuda, Org. Synth. 1988, 66, 142-150. http://dx.doi.org/10.15227/orgsyn.066.0142

5. R. M. Mohareb, K. El-Sharkawy, S. M. Sherif, Acta Pharm. 2008, 58, 429-444.

6. A. M. Salah, Heteroatom Chem. 2003, 14, 612-614. http://dx.doi.org/10.1002/hc.10199

7. P. A. Crooks, L. P. Dwoskin, Biochemical Pharmacology 1997, 54, 743-753. http://dx.doi.org/10.1016/S0006-2952(97)00117-2

8. B. K. Warren, E. E. Knaus, Eur. J. Med. Chem. 1987, 22, 411-415. http://dx.doi.org/10.1016/0223-5234(87)90028-6

9. B. J. Mulchin, C. G. Newton, J. W. Baty, C. H. Grasso, W. J. Martin, M. C. Walton, E. M. Dangerfield, C. H. Plunkett, M. V. Berridge, J. L. Harper, M. S. Timmer, B. L. Stocker, Bioorg. Med. Chem. 2010, 18, 3238-3251. http://dx.doi.org/10.1016/j.bmc.2010.03.021

10. Y.-L. Luo, K. Baathulaa, V. K. Kannekanti, C.-H. Zhou, G.X. Cai, Sci. China Chem. 2015, 58, 483-494. http://dx.doi.org/10.1007/s11426-014-5296-3

11. J. N. Sangshetti, P. P. Dharmadhikari, R. S. Chouthe, B. Fatema, V. Lad, V. Karande, S. N. Darandale, D. B. Shinde, Bioorg. Med. Chem. Lett. 2013, 23, 2250-2253. http://dx.doi.org/10.1016/j.bmcl.2013.01.041

12. J. Witherington, V. Bordas, S. L. Garland, D. M. B. Hickey, R. J. Ife, J. Liddle, M. Saunders, D. G. Smith, R. W. Ward, Bioorg. Med. Chem. Lett. 2003, 13, 1577-1580. http://dx.doi.org/10.1016/S0960-894X(03)00134-3

13. X.-H. Liu, H.-F. Liu, X. Shen, B.-A. Song, P. S. Bhadury, H.L. Zhu, J.-X. Liu, X.-B. Qi, Bioorg. Med. Chem. Lett. 2010 , 20, 4163-4167. http://dx.doi.org/10.1016/j.bmcl.2010.05.080

14. S. Ram, Progress in Medicinal Chemistry 1988, 25, 233-247. http://dx.doi.org/10.1016/S0079-6468(08)70279-0

15. M. Gaba, S. Singh, C. Mohan, Eur. J. Med. Chem. 2014, 76, 494-505. http://dx.doi.org/10.1016/j.ejmech.2014.01.030

16. Y. M. Shaker, M. A. Omar, K. Mahmoud, S. M. Elhallouty, W. M. El-Senousy, M. M. Ali, A. E. Mahmoud, A. H. AbdelHalim, S. M. Soliman, H. I. El Diwani, J. Enzyme Inhib. Med. Chem. 2015, 8, 1-20.

17. J. J. B. Nevado, G. C. Peñalvo, R. M. R. Dorado, V. R. Robledo, J. Pharm. Biomed. Anal. 2014, 92, 211-219. http://dx.doi.org/10.1016/j.jpba.2013.12.020

18. S. Fujimoto, Eur. J. Pharm. 1994, 265, 159-166. http://dx.doi.org/10.1016/0014-2999(94)90426-X

19. A. D. Khoje, C. Charnock, B. Wan, S. Franzblau, L.-L. Gundersen, Bioorg. Med. Chem. 2011, 19, 3483-3491. http://dx.doi.org/10.1016/j.bmc.2011.04.023

20. D. Seenaiah, P. R. Reddy, G. M. Reddy, A. Padmaja, V. Padmavathi, N. S. Krishna, Eur. J. Med. Chem. 2014, 77, 1-7. http://dx.doi.org/10.1016/j.ejmech.2014.02.050

21. E. C. Herrmann, J. A. Herrmann, D. C. Delong, Antiviral Research, 1981, 1, 301-314. http://dx.doi.org/10.1016/0166-3542(81)90022-X

22. S. Sharma, S. Gangal, A. Rauf, Eur. J. Med. Chem. 2009, 44, 1751-1757. http://dx.doi.org/10.1016/j.ejmech.2008.03.026

23. M. Rashid, A. Husain, R. Mishra, Eur. J. Med. Chem. 2012, 54, 855-866.

http://dx.doi.org/10.1016/j.ejmech.2012.04.027

24. R. S. Harapanhalli, R. W. Howell, D. V. Rao, Nuclear Med. Biol. 1994, 21, 641-647. http://dx.doi.org/10.1016/0969-8051(94)90030-2

25. S. Singh, B. S. Dwarakanath, T. L. Mathew, J. Phtotochem. Photobiol. B. 2004, 77, 45-54. http://dx.doi.org/10.1016/S1011-1344(04)00122-8

26. H. A. S. Elzahabi, Eur. J. Med. Chem. 2011, 46, 4025-4034. http://dx.doi.org/10.1016/j.ejmech.2011.05.075

27. J. R. Tseng, D. Stuart, K. Aardalen, A. Kaplan, N. Aziz, N. P. Hughes, S. S. Gambhir, Neoplasia 2011, 13, 266-275. http://dx.doi.org/10.1593/neo.101466

28. T. R. Reddy, G. R. Reddy, L. S. Reddy, S. Jammula, Y. Lingappa, R. Kapavarapu, C. L. Meda, K. V. Parsa, M. Pal, Eur. J. Med. Chem. 2012, 48, 265-274. http://dx.doi.org/10.1016/j.ejmech.2011.12.024

29. S. Khaksar, M. Yaghoobi, J. Fluor. Chem. 2012, 142, 41-44. http://dx.doi.org/10.1016/j.jfluchem.2012.06.009

30. R. M. Mohareb, S. M. Sherif, Arch. Die Pharm. (Weinheim), 1991, 324, 469-471.

http://dx.doi.org/10.1002/ardp.2503240801 
31. R. M. Mohareb, N. I. Abdel-Sayed, S. M. Sherif, Phosphorus, Sulfur, Silicon Relat. Elem. 1991, 63, 119-129. http://dx.doi.org/10.1080/10426509108029435

32. R. M. Mohareb, Monatsh. Chem. 1992, 123, 341-347. http://dx.doi.org/10.1007/BF00810946

33. J. Chodosh, R. D. Dix, R. C. Howell, W. G. Stroop, S. C. G. Tseng, Invest. Ophthalmol. Vis. Sci. 1994, 35, 1046-1058.

34. A. U. Rahman, M. I. Choudhary, W. J. Thomsen, Bioassay Techniques for Drug Development, Harwood Academic, Amsterdam, The Netherlands, 2001. http://dx.doi.org/10.4324/9780203304532

35. B. Brayn, M. Timothy, S. Tore, General and Applied Toxicology, $2^{\text {nd }}$ ed., vol. I, $52 \mathrm{p}$.

36. J. L. Carballo, Z. L. H. Inda, P. Pérez, M. D. G. Grávalos, BMC Biotechnol. 2002, 2, 17-22.

http://dx.doi.org/10.1186/1472-6750-2-17

37. M. Calleja, G. Persoone, Atla. 1992, 20, 396-405.

38. P. N. Chatterjee, S. Roy, Tetrahedron 2011, 67, 4569-4577. http://dx.doi.org/10.1016/j.tet.2011.04.092

\section{Povzetek}

Pri reakciji etil cianoacetat z $o$-fenilendiaminom je nastal 2-cianometilbenzo[c]imidazol (1). To spojino smo uporabili kot ključno izhodno snov za sintezo biološko aktivnih heterocikličnih derivatov. Pri reakciji $1 \mathrm{~s}$ cikloheksanonom ter benzaldehidom, 4-metoksibenzaldehidom oz. 4-klorobenzaldehidom so nastali pripojeni derivati 2a-c. Testiranje antitumorske aktivnosti novopripravljenih produktov proti trem rakastim celičnim linijam, t.j. MCF-7 (adeno-carcinom dojke), NCI-H460 (nemikrocelični karcinom pljuč) in SF-268 (rak centralnega živčnega sistema), je pokazalo, da spojine

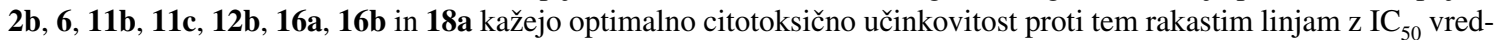
nostmi v nM območju. Ker so bioaktivne spojine pogosto strupene za ličinke morskih rakcev, smo se odločili preveriti še in vivo strupenost teh spojin na ličinke Artemia salina. Spojine 11b, 12b in $\mathbf{1 6 b}$ niso pokazale nobene strupenosti za testirane organizme. 